Pacific

Journal of

Mathematics

\title{
HILBERT SERIES OF CERTAIN JET SCHEMES OF DETERMINANTAL VARIETIES
}

Sudhir R. GHORPade, Boyan Jonov AND B. A. SETHuraman 


\title{
HILBERT SERIES OF CERTAIN JET SCHEMES OF DETERMINANTAL VARIETIES
}

\author{
SudHIR R. GHORpade, BOYAN JONOV AND B. A. SeThuRAMAN
}

\begin{abstract}
We consider the affine variety $\mathscr{E}_{2,2}^{m, n}$ of first-order jets over $\mathscr{E}_{2}^{m, n}$, where $\mathscr{E}_{2}^{m, n}$ is the classical determinantal variety given by the vanishing of all $2 \times 2$ minors of a generic $m \times n$ matrix. When $2<m \leq n$, this jet scheme $\mathscr{L}_{2,2}^{m, n}$ has two irreducible components: a trivial component, isomorphic to an affine space, and a nontrivial component that is the closure of the jets supported over the smooth locus of $\mathscr{F}_{2}^{m, n}$. This second component is referred to as the principal component of $\mathscr{F}_{2,2}^{m, n}$; it is, in fact, a cone and can also be regarded as a projective subvariety of $\mathbb{P}^{2 m n-1}$. We prove that the degree of the principal component of $\mathscr{E}_{2,2}^{m, n}$ is the square of the degree of $\mathscr{E}_{2}^{m, n}$ and, more generally, the Hilbert series of the principal component of $\mathscr{E}_{2,2}^{m, n}$ is the square of the Hilbert series of $\mathscr{E}_{2}^{m, n}$. As an application, we compute the $a$-invariant of the principal component of $\mathscr{E}_{2,2}^{m, n}$ and show that the principal component of $\mathscr{E}_{2,2}^{m, n}$ is Gorenstein if and only if $m=n$.
\end{abstract}

\section{Introduction}

Let $\mathbb{F}$ be an algebraically closed field and $m, n, r$ be integers with $1 \leq r \leq m \leq n$. Let $\mathscr{E}_{r}^{m, n}$ denote the affine variety in $\mathbb{A}_{\mathbb{F}}^{m n}$ defined by the vanishing of all $r \times r$ minors of an $m \times n$ matrix whose entries are independent indeterminates over $\mathbb{F}$. Equivalently $\mathscr{E}_{r}^{m, n}$ is the locus of $m \times n$ matrices over $\mathbb{F}$ of rank $<r$. This is a classical and well-studied object and a number of its properties are known. For example, we know that $\mathscr{L}_{r}^{m, n}$ is irreducible, rational, arithmetically Cohen-Macaulay and projectively normal. Moreover the multiplicity of $\mathscr{L}_{r}^{m, n}$ (at its vertex, since $\mathscr{L}_{r}^{m, n}$ is evidently a cone) or, equivalently, the degree of the corresponding projective subvariety of $\mathbb{P}_{\mathbb{F}}^{m n-1}$ is given by the following elegant formula (see [Abhyankar 1988, Remarks 20.18 and 20.19] or [Ghorpade 1994, Corollary 6.2]; see also [Herzog and Trung 1992] for an alternative proof and [Arbarello et al. 1985, Chapter 2, §4] or [Ghorpade and Krattenthaler 2004, p. 352] for an alternative approach and a different formula): 


$$
e\left(\mathscr{E}_{r}^{m, n}\right)=\operatorname{det}_{1 \leq i, j \leq r-1}\left(\left(\begin{array}{c}
m+n-i-j \\
m-i
\end{array}\right)\right) .
$$

More generally, the Hilbert series of $\mathscr{E}_{r}^{m, n}$ (or, more precisely, of the corresponding projective subvariety of $\mathbb{P}_{\mathbb{F}}^{m n-1}$ ) is also known and is explicitly given by

$$
\frac{\sum_{k \geq 0} h_{k} t^{k}}{(1-t)^{d}}
$$

where $d=(r-1)(m+n-r+1)$ is the dimension of $\mathscr{E}_{r}^{m, n}$ (as an affine variety), and the coefficients $h_{k}$ are given by sums of binomial determinants as follows:

$$
h_{k}=\sum_{k_{1}+\cdots+k_{r-1}=k} \operatorname{det}_{1 \leq i, j \leq r-1}\left(\left(\begin{array}{c}
m-i \\
k_{i}
\end{array}\right)\left(\begin{array}{c}
n-j \\
k_{i}+i-j
\end{array}\right)\right) \text {. }
$$

For a proof of this formula, we refer to [Ghorpade 1996] (see also [Galligo 1985] and [Conca and Herzog 1994]). Using this, or otherwise (see [Svanes 1974]), it can be shown that $\mathscr{E}_{r}^{m, n}$ is Gorenstein if and only if $m=n$. Moreover one can also show that the $a$-invariant of the (homogeneous) coordinate ring of $\mathscr{E}_{r}^{m, n}$ (which, by definition, is the least degree of a generator of its graded canonical module) is $n(1-r)$; see, e.g., [Gräbe 1988] or [Ghorpade 1996, Theorem 4].

We now turn to jet schemes, which have been of much recent interest due in large part to Nash's suggestion [1995] that jet schemes should give information about singularities of the base; see, e.g., [Mustaţă 2001; 2002; Ein and Mustaţă 2009]. If $\mathscr{E}$ is a scheme of finite type over $\mathbb{F}$ and $k$ a positive integer, then a $(k-1)$-jet on $\mathscr{E}$ is a morphism $\operatorname{Spec} \mathbb{F}[t] /\left(t^{k}\right) \rightarrow \mathscr{L}$. The set of $(k-1)$-jets on $\mathscr{E}$ forms a scheme of finite type over $\mathbb{F}$, denoted $\mathscr{F}_{k-1}(\mathscr{L})$ and called the $(k-1)$-th jet scheme of $\mathscr{L}$. A little more concretely, suppose $\mathscr{L}$ is the affine scheme Spec $S / I$ defined by the ideal $I=\left\langle f_{1}, \ldots, f_{s}\right\rangle$ in the polynomial ring $S=\mathbb{F}\left[X_{1}, \ldots, X_{N}\right]$. Consider independent indeterminates $t$ and $X_{i}^{(\ell)}(i=1, \ldots, N$ and $\ell=0, \ldots, k-1)$ over $\mathbb{F}$ and the corresponding polynomial ring $S^{(k)}$ in the $N k$ variables $X_{i}^{(\ell)}$. For each $j=1, \ldots, s$, the polynomial

$$
f_{j}\left(X_{1}^{(0)}+t X_{1}^{(1)}+\cdots+t^{k-1} X_{1}^{(k-1)}, \ldots, X_{N}^{(0)}+t X_{N}^{(1)}+\cdots+t^{k-1} X_{N}^{(k-1)}\right)
$$

is of the form

$$
f_{j}^{(0)}+t f_{j}^{(1)}+\cdots+t^{k-1} f_{j}^{(k-1)} \quad \text { modulo }\left\langle t^{k}\right\rangle
$$

for unique $f_{j}^{(\ell)} \in S^{(k)}(0 \leq \ell<k)$. Then $\mathscr{F}_{k-1}(\mathscr{L})$ is the affine scheme $\operatorname{Spec} S^{(k)} / I^{\prime}$, where $I^{\prime}$ is the ideal generated by all $f_{j}^{(\ell)}, 1 \leq j \leq s, 0 \leq \ell<k$, (Often in the literature, authors conflate the algebraic set in $\mathbb{A}^{N k}$ consisting of the zeros of the polynomials $f_{j}^{(\ell)}$ with $\mathscr{F}_{k-1}(\mathscr{L})$ itself. This is generally harmless, especially when considering topological properties such as components, since the points of this 
algebraic set correspond bijectively with the set of closed points of $\mathscr{I}_{k-1}(\mathscr{L})$ as $\mathbb{F}$ is algebraically closed, and the set of closed points of an affine scheme is dense in the scheme. See [Liu 2002, Chapter 2, Remark 3.49], for instance.)

When $\mathscr{L}$ is smooth of dimension $d$, the jet scheme $\mathscr{F}_{k-1}(\mathscr{L})$ is known to be smooth of dimension $k d$. In general, $\mathscr{F}_{k-1}(\mathscr{L})$ can have multiple irreducible components, and these include a principal component that corresponds to the closure of the set of jets supported over the smooth points of the base scheme $\mathscr{L}$. These components are usually quite complicated and interesting. In fact, very little seems to be known about the structure of these components and their numerical invariants such as multiplicities. For example, even when $\mathscr{E}$ is a monomial scheme such as the one given by $X_{1} X_{2} \cdots X_{e}=0$, where $e \leq N$, determining the irreducible components and the multiplicity of $\mathscr{F}_{k-1}(\mathscr{E})$ appears to require some effort; see, e.g., [Goward and Smith 2006] and [Yuen 2007b]. Irreducible components of jet schemes of toric surfaces are discussed in [Mourtada 2011], while the irreducibility of jet schemes of the commuting matrix pairs scheme is discussed in [Sethuraman and Šivic 2009]. In a more recent work [Bruschek et al. 2011], the Hilbert series of arc spaces (that are, in a sense, limits of $k$-th jet schemes as $k \rightarrow \infty$ ) of seemingly simple objects such as the double line $y^{2}=0$ are shown to have connections with the Rogers-Ramanujan identities.

Now determinantal varieties such as $\mathscr{E}_{r}^{m, n}$ above are natural examples of singular algebraic varieties, and it is not surprising that the study of their jet schemes has been of considerable interest. This was done first by Košir and Sethuraman [2005a; 2005b] (see also [Yuen 2007a]). To describe the related results, henceforth we fix positive integers $r, k, m, n$ with $r \leq m \leq n$, and let $\mathscr{E}_{r, k}^{m, n}$ denote the $(k-1)$-th jet scheme on $\mathscr{E}_{r}^{m, n}$. It was shown in [Košir and Sethuraman 2005a] that $\mathscr{E}_{r, k}^{m, n}$ is irreducible of codimension $k(n-m+1)$ when $r=m$, and if $r<m$, then it can have $\geq 1+\lfloor k / 2\rfloor$ irreducible components with equality when $r=2$ or $k=2$. A more unified result was obtained in [Docampo 2013], showing that $\mathscr{E}_{r, k}^{m, n}$ has exactly $k+1-\lceil k / r\rceil$ irreducible components. At any rate, the best understood case with multiple components is $\mathscr{E}_{2,2}^{m, n}$, where $2<m \leq n$. In this case $\mathscr{L}_{2,2}^{m, n}=Z_{0} \cup Z_{1}$, where $Z_{1}$ is isomorphic to $\mathbb{A}^{m n}$ while $Z_{0}$ is the principal component which is the closure of the jets supported over the smooth points of the base variety $\mathscr{E}_{2}^{m, n}$. Here it will be convenient to consider $2 m n$ indeterminates, denoted $x_{i, j}, y_{i, j}$ for $1 \leq i \leq m, 1 \leq j \leq n$, and the corresponding polynomial ring $R=\mathbb{F}\left[x_{i, j}, y_{i, j}: 1 \leq i \leq m, 1 \leq j \leq n\right]$. Also let $\mathscr{I}=\mathscr{I}_{2,2}^{m, n}$ and $\mathscr{I}_{0}$ denote, respectively, the ideals of $R$ corresponding to the jet scheme $\mathscr{E}_{2,2}^{m, n}$ and its principal component $Z_{0}$. In [Košir and Sethuraman 2005b], it was shown that both $\mathscr{I}$ and $\mathscr{I}_{0}$ are homogeneous radical ideals of $R$ (so that $\mathscr{I}_{0}$ is prime), and moreover their Gröbner bases were explicitly determined. The leading term ideal LT( $\left.\mathscr{I}_{0}\right)$ of $\mathscr{I}_{0}$ with respect to this Gröbner basis is generated by squarefree monomials and hence $R / \operatorname{LT}\left(\mathscr{I}_{0}\right)$ is the Stanley-Reisner ring of a simplicial complex $\Delta_{0}$. Jonov [2011] 
subsequently studied this simplicial complex. He showed that $\Delta_{0}$ is shellable and thus deduced that $R / \mathscr{I}_{0}$ is Cohen-Macaulay. (This last result was independently obtained in [Smith and Weyman 2007] as well, using a geometric technique for computing syzygies.) Jonov also found a formula for the multiplicity of $R / \Phi_{0}$, namely,

$$
e\left(R / \mathscr{I}_{0}\right)=\sum_{\substack{i=1 \\
(i, j) \neq(m, n)}}^{m} \sum_{j=1}^{n}\left(\begin{array}{c}
m+n-i-j \\
m-i
\end{array}\right) \operatorname{det}\left(\begin{array}{cc}
\left(\begin{array}{c}
i+n-2 \\
i-1
\end{array}\right) & \left(\begin{array}{c}
m+j-2 \\
m-1
\end{array}\right) \\
\left(\begin{array}{c}
i+n-3 \\
i-2
\end{array}\right) & \left(\begin{array}{c}
m+j-3 \\
m-2
\end{array}\right)
\end{array}\right) .
$$

Equation (3) above is the starting point of the present paper. We first show that the right side of this equation simplifies remarkably to yield the pretty result

$$
e\left(R / I_{0}\right)=\left(\begin{array}{c}
m+n-2 \\
m-1
\end{array}\right)^{2}=e\left(\mathscr{L}_{2}^{m, n}\right)^{2} .
$$

(this was already alluded to in [Jonov 2011, Remark 2.8]). Next we proceed to determine the Hilbert series of $R / \mathscr{I}_{0}$ or of the principal component $Z_{0}$. We use the well-known connections between the Hilbert series of $R / \Phi_{0}$, that of $R / \operatorname{LT}\left(\Phi_{0}\right)$ and the shelling of the facets of the simplicial complex $\Delta_{0}$ obtained in [Jonov 2011]. With some effort we are led to an initial formula for the Hilbert series of $R / \mathscr{I}_{0}$, which is enormously complicated and involves multiple sums of products of binomials in the same vein as the right side of (3). But we persist with the combinatorics and are eventually rewarded with the main result of this paper. Namely, just like the multiplicity, the Hilbert series of $R / \mathscr{I}_{0}$ is precisely the square of the Hilbert series of the base determinantal variety $\mathscr{E}_{2}^{m, n}$. As a corollary of this, we are able to determine the $a$-invariant of $R / \mathscr{I}_{0}$ and the Hilbert series of its graded canonical module. Moreover we show that, as in the case of classical determinantal varieties, $Z_{0}$ is Gorenstein if and only if $m=n$.

The proofs given here are completely elementary but highly combinatorial and rather intricate. Heuristically it appears to us that up to some flat deformation (such as the Gröbner deformation of $\Phi_{0}$ to $\operatorname{LT}\left(\Phi_{0}\right)$, which preserves the Hilbert series), the coordinate ring of the principal component (suitably deformed) should look like the tensor product of the coordinate ring of the base (similarly deformed) with itself. (This would reflect the fact that, at the smooth points, the base variety locally looks like its tangent space.) It would follow then that the Hilbert series of the principal component is the square of that of $\mathscr{E}_{2}^{m, n}$. We emphasize that this is only heuristics (with all of its ever-present dangers); nevertheless we suspect that analogous results relating the Hilbert series of the principal component to that of the base scheme should hold more generally for all $\mathscr{E}_{r, k}^{m, n}$, and possibly also for jet schemes over a wider class of affine base schemes. We do not know how to prove this, and leave it open for investigation. 


\section{Binomials and lattice paths}

In this section we collect some preliminaries concerning binomial coefficients, alterations of summations, and lattice paths. These will be useful in the sequel.

2.1. Binomials. To begin with, let us recall that the binomial coefficient $\left(\begin{array}{l}s \\ a\end{array}\right)$ is defined for any integer parameters $s, a$ (and with the standard convention that the empty product is taken as 1) as follows:

$$
\left(\begin{array}{l}
s \\
a
\end{array}\right)= \begin{cases}\frac{s(s-1) \cdots(s-a+1)}{a !} & \text { if } a \geq 0, \\
0 & \text { if } a<0 .\end{cases}
$$

In fact, this definition makes sense not only for any $s \in \mathbb{Z}$ but also for $s$ in any overring of $\mathbb{Z}$ and in particular, $s$ can be an indeterminate over $\mathbb{Q}$ in which case $\left(\begin{array}{l}s \\ a\end{array}\right)$ is a polynomial in $s$ of degree $a$, provided $a \geq 0$. Now let $s, a \in \mathbb{Z}$. Note that

$$
\left(\begin{array}{l}
s \\
a
\end{array}\right)=0 \Longleftrightarrow \text { either } a<0 \text { or } a>s \geq 0 .
$$

One has to be careful with the validity of some of the familiar identities; for example,

$$
\left(\begin{array}{l}
s \\
a
\end{array}\right)=\left(\begin{array}{c}
s \\
s-a
\end{array}\right) \Longleftrightarrow \text { either } s \geq 0 \text { or } s<a<0,
$$

whereas some standard identities such as the Pascal triangle identity or its alternative equivalent version below are valid for arbitrary integer parameters:

$$
\left(\begin{array}{c}
s \\
a-1
\end{array}\right)+\left(\begin{array}{c}
s \\
a
\end{array}\right)=\left(\begin{array}{c}
s+1 \\
a
\end{array}\right) \text { and }\left(\begin{array}{c}
s+a \\
a
\end{array}\right)+\left(\begin{array}{c}
s+a \\
a+1
\end{array}\right)=\left(\begin{array}{c}
s+a+1 \\
a+1
\end{array}\right) .
$$

The equivalence of the two identities above follows from the simple fact below, which is also valid for arbitrary integer parameters:

$$
\left(\begin{array}{c}
s+a \\
a
\end{array}\right)=(-1)^{a}\left(\begin{array}{c}
-s-1 \\
a
\end{array}\right), \quad \text { that is, } \quad\left(\begin{array}{l}
s \\
a
\end{array}\right)=(-1)^{a}\left(\begin{array}{c}
a-s-1 \\
a
\end{array}\right) .
$$

We now record some basic facts, which are often used in later sections. Proofs are easy and are briefly outlined for the sake of completeness.

Lemma 1. For any e, $s, t \in \mathbb{Z}$ with $s \leq t$, we have

$$
\sum_{s<d \leq t}\left(\begin{array}{l}
d \\
e
\end{array}\right)=\left(\begin{array}{c}
t+1 \\
e+1
\end{array}\right)-\left(\begin{array}{c}
s+1 \\
e+1
\end{array}\right) .
$$

Proof. Induct on $t-s$, using the first identity in (6) to rewrite $\left(\begin{array}{c}t+1 \\ e+1\end{array}\right)$.

The following result is a version of the so-called Chu-Vandermonde identity. 
Lemma 2. For any $s, t, \alpha, \beta \in \mathbb{Z}$, we have

$$
\sum_{j \in \mathbb{Z}}\left(\begin{array}{c}
s \\
\alpha+j
\end{array}\right)\left(\begin{array}{c}
t \\
\beta-j
\end{array}\right)=\left(\begin{array}{c}
s+t \\
\alpha+\beta
\end{array}\right)
$$

and

$$
\sum_{j \in \mathbb{Z}}\left(\begin{array}{c}
s+\alpha+j \\
\alpha+j
\end{array}\right)\left(\begin{array}{c}
t+\beta-j \\
\beta-j
\end{array}\right)=\left(\begin{array}{c}
s+t+\alpha+\beta+1 \\
\alpha+\beta
\end{array}\right),
$$

where, in view of (4), the summation on the left in (8) as well as in (9) is essentially finite in the sense that all except finitely many summands are zero.

Proof. Let $X$ be an indeterminate over $\mathbb{Q}$. Use the binomial theorem, namely,

$$
(1+X)^{d}=\sum_{i=0}^{\infty}\left(\begin{array}{l}
d \\
i
\end{array}\right) X^{i},
$$

which is valid in the formal power series ring $\mathbb{Q} \llbracket X \rrbracket$ for any $d \in \mathbb{Z}$, and compare the coefficients of $X^{\alpha+\beta}$ on the two sides of the identity $(1+X)^{s}(1+X)^{t}=(1+X)^{s+t}$ to obtain (8). Now (8) and (7) imply (9).

2.2. Alterations of summations. As in (8) and (9) above, we will often deal with summations that are essentially finite, by which we mean that the parameters in the sum range over an infinite set, but the summand is zero for all except finitely many values of parameters, and so the summation is, in fact, finite. It is, however, very useful that the parameters range freely over a seemingly infinite set so that useful alterations such as the ones listed below can be readily made. These are too obvious to be stated as lemmas and proved formally. But for ease of reference, we record below some elementary transformations of essentially finite summations. In what follows, $f: \mathbb{Z}^{2} \rightarrow \mathbb{Q}$ will denote a rational-valued function of two integer parameters with the property that the support of $f$, namely, the set $\left\{\left(s_{1}, s_{2}\right) \in \mathbb{Z}^{2}: f\left(s_{1}, s_{2}\right) \neq 0\right\}$ is finite or more generally, it is diagonally finite, that is, for each $k \in \mathbb{Z}$, the set $\left\{\left(s_{1}, s_{2}\right) \in \mathbb{Z}^{2}: s_{1}+s_{2}=k\right.$ and $\left.f\left(s_{1}, s_{2}\right) \neq 0\right\}$ is finite. In this case, for any $v \in \mathbb{Z}$ and any $\alpha, \beta \in \mathbb{Z}$ such that $\alpha+\beta=v$, we have

$$
\sum_{s_{1}+s_{2}=k-v} f\left(s_{1}, s_{2}\right)=\sum_{t_{1}+t_{2}=k} f\left(t_{1}-\alpha, t_{2}-\beta\right),
$$

where writing $s_{1}+s_{2}=k-v$ below the first summation indicates that the sum is over all $\left(s_{1}, s_{2}\right) \in \mathbb{Z}^{2}$ satisfying $s_{1}+s_{2}=k-v$. A similar meaning applies for the second summation and in fact, for all such summations appearing in the sequel. Since the "diagonal condition" $t_{1}+t_{2}=k$ is symmetric, we also have

$$
\sum_{t_{1}+t_{2}=k} f\left(t_{1}, t_{2}\right)=\sum_{t_{1}+t_{2}=k} f\left(t_{2}, t_{1}\right)
$$


Figure 1. A lattice path from $A=(1,1)$ to $E=(4,5)$.

Thus, for example, using (10) and (11), we find

$$
\sum_{t_{1}+t_{2}=k} f\left(t_{1}, t_{2}\right)=\sum_{t_{1}+t_{2}=k} f\left(t_{2}+1, t_{1}-1\right)=\sum_{t_{1}+t_{2}=k} f\left(t_{1}+1, t_{2}-1\right) .
$$

2.3. Lattice paths. Let $A=\left(a, a^{\prime}\right)$ and $E=\left(e, e^{\prime}\right)$ be points in the integer lattice $\mathbb{Z}^{2}$. By a lattice path from $A$ to $E$ we mean a finite sequence $L=\left(P_{0}, P_{1}, \ldots, P_{t}\right)$ of points in $\mathbb{Z}^{2}$ with $P_{0}=A, P_{t}=E$ and

$$
P_{i}-P_{i-1}=(1,0) \text { or }(0,1) \text { for } i=1, \ldots, t .
$$

The lattice path $L$ can and will be identified with its point set $\left\{P_{j}: 0 \leq j \leq t\right\}$; indeed $L$ is obtained by simply arranging the elements of this set in a lexicographic order. The point $A=P_{0}$ is called the initial point of $L$ while $E=P_{t}$ is called the end point of $L$. We say that a point $P_{j}$ is a NE-turn of the lattice path $L$ if $0<j<t$ and $P_{j}-P_{j-1}=(0,1)$ while $P_{j+1}-P_{j}=(1,0)$. Note that a lattice path is also determined by its NE turns.

In more intuitive terms, a lattice path consists of vertical or horizontal steps of length 1, and a NE-turn is simply a northeast turn. For example, a lattice path from $A=(1,1)$ to $E=(4,5)$ may be depicted as in Figure 1, and it may be noted that the points $(1,2)$ and $(2,4)$ are its NE turns.

If we let $\mathscr{P}(A \rightarrow E)$ denote the set of lattice paths from $A=\left(a, a^{\prime}\right)$ to $E=\left(e, e^{\prime}\right)$ and, for any $k \in \mathbb{Z}$, let $\mathscr{P}_{k}(A \rightarrow E)$ denote the subset of $\mathscr{P}(A \rightarrow E)$ consisting of lattice paths with exactly $k$ NE turns, then it is easily seen that

$$
\begin{gathered}
|\mathscr{P}(A \rightarrow E)|=\left(\begin{array}{c}
e-a+e^{\prime}-a^{\prime} \\
e-a
\end{array}\right), \\
\left|\mathscr{P}_{k}(A \rightarrow E)\right|=\left(\begin{array}{c}
e-a \\
k
\end{array}\right)\left(\begin{array}{c}
e^{\prime}-a^{\prime} \\
k
\end{array}\right),
\end{gathered}
$$

where as usual, for a finite set $\mathscr{P}$, we denote by $|\mathscr{P}|$ the cardinality of $\mathscr{P}$. Given any two $d$-tuples $\mathscr{A}=\left(A_{1}, \ldots, A_{d}\right)$ and $\mathscr{E}=\left(E_{1}, \ldots, E_{d}\right)$ of points in $\mathbb{Z}^{2}$, by a lattice path from $\mathscr{A}$ to $\mathscr{E}$ we mean a $d$-tuple $\mathscr{L}=\left(L_{1}, \ldots, L_{d}\right)$, where $L_{r}$ is a lattice path from $A_{r}$ to $E_{r}$, for $1 \leq r \leq d$. We call $\mathscr{L}$ to be nonintersecting if no 
two of the paths $L_{1}, \ldots, L_{d}$ have a point in common. We say that $\mathscr{L}$ has $k \mathrm{NE}$ turns if the total number of NE turns in the $d$ paths $L_{1}, \ldots, L_{d}$ is $k$. The set of nonintersecting lattice paths from $\mathscr{A}=\left(A_{1}, \ldots, A_{d}\right)$ to $\mathscr{E}=\left(E_{1}, \ldots, E_{d}\right)$ will be denoted by $\mathscr{P}\left(A_{1} \rightarrow E_{1}, \ldots, A_{d} \rightarrow E_{d}\right)$ or simply by $\mathscr{P}(\mathscr{A} \rightarrow \mathscr{E})$, and its subset consisting of nonintersecting lattice paths with exactly $k$ NE turns will be denoted by $\mathscr{P}_{k}\left(A_{1} \rightarrow E_{1}, \ldots, A_{d} \rightarrow E_{d}\right)$ or simply by $\mathscr{P}_{k}(\mathscr{A} \rightarrow \mathscr{E})$.

Proposition 3. Let $d$ be a positive integer and let $A_{r}=\left(a_{r}, a_{r}^{\prime}\right)$ and $E_{r}=\left(e_{r}, e_{r}^{\prime}\right)$, $r=1, \ldots, d$, be points in $\mathbb{Z}^{2}$. Also let $\mathscr{A}=\left(A_{1}, \ldots, A_{d}\right)$ and $\mathscr{E}=\left(E_{1}, \ldots, E_{d}\right)$.

(i) Suppose

$$
a_{1} \leq \cdots \leq a_{d}, \quad e_{1} \leq \cdots \leq e_{d} \quad \text { and } \quad a_{1}^{\prime} \geq \cdots \geq a_{d}^{\prime}, \quad e_{1}^{\prime} \geq \cdots \geq e_{d}^{\prime} .
$$

Then the number of nonintersecting lattice paths from $\mathscr{A}$ to $\mathscr{E}$ is equal to

$$
\operatorname{det}\left(\left(\begin{array}{c}
e_{j}-a_{i}+e_{j}^{\prime}-a_{i}^{\prime} \\
e_{j}-a_{i}
\end{array}\right)_{1 \leq i, j \leq d}\right)
$$

(ii) Let $k \in \mathbb{Z}$ and suppose

$$
a_{1} \leq \cdots \leq a_{d}, \quad e_{1}<\cdots<e_{d} \text { and } a_{1}^{\prime}>\cdots>a_{d}^{\prime}, \quad e_{1}^{\prime} \geq \cdots \geq e_{d}^{\prime} .
$$

Then the number of nonintersecting lattice paths from $A$ to $\mathscr{E}$ with exactly $k$ NE turns is equal to

$$
\sum_{k_{1}+\cdots+k_{d}=k} \operatorname{det}\left(\left(\begin{array}{c}
e_{j}-a_{i}+i-j \\
k_{i}+i-j
\end{array}\right)\left(\begin{array}{c}
e_{j}^{\prime}-a_{i}^{\prime}-i+j \\
k_{i}
\end{array}\right)_{1 \leq i, j \leq d}\right)
$$

Part (i) of the above proposition is due to Gessel and Viennot [1985, Theorem 1], although some of the ideas can be traced back to Chaundy [1932], Karlin and McGregor [1959], and Lindström [1973]. The statement here is a little more general than that of [Gessel and Viennot 1985], and a proof can be found, for example, in [Ghorpade 2001, §3] or [Krattenthaler 1995b, §2.2]. Part (ii) was proved independently by Modak [1992], Krattenthaler [1995a] and Kulkarni [1996] (see also [Ghorpade 1996]), although the hypothesis in [Modak 1992] and [Kulkarni 1996] on the coordinates of the initial and the end points is slightly more restrictive than in (ii) above where we follow [Krattenthaler 1995a, Theorem 1]. The following consequence is frequently used in Section 4.

Corollary 4. For any $a, b, c, d, s \in \mathbb{Z}$ with $a<c$ and $b \geq d$, the cardinality of $\mathscr{P}_{s}((1,2) \rightarrow(a, b),(1,1) \rightarrow(c, d))$ is given by

$$
\sum_{s_{1}+s_{2}=s}\left(\begin{array}{c}
a-1 \\
s_{1}
\end{array}\right)\left(\begin{array}{c}
b-2 \\
s_{1}
\end{array}\right)\left(\begin{array}{c}
c-1 \\
s_{2}
\end{array}\right)\left(\begin{array}{c}
d-1 \\
s_{2}
\end{array}\right)-\left(\begin{array}{c}
a \\
s_{2}+1
\end{array}\right)\left(\begin{array}{c}
b-2 \\
s_{2}
\end{array}\right)\left(\begin{array}{c}
c-2 \\
s_{1}-1
\end{array}\right)\left(\begin{array}{c}
d-1 \\
s_{1}
\end{array}\right) .
$$

Proof. This is just a special case of part (ii) of Proposition 3. 


\section{Multiplicity}

As in the Introduction, we fix in the remainder of this paper an algebraically closed field $\mathbb{F}$ and integers $m, n$ with $2<m \leq n$. Also let $x_{i, j}, y_{i, j}, 1 \leq i \leq m, 1 \leq j \leq n$, be independent indeterminates over $\mathbb{F}$. Denote by $V_{x}$ the set

$$
\left\{x_{i, j}: 1 \leq i \leq m \text { and } 1 \leq j \leq n\right\}
$$

of the " $x$-variables", and by $V_{y}$ a similar set of the " $y$-variables". Let $V=V_{x} \cup V_{y}$ and let $R=\mathbb{F}[V]$ be the corresponding polynomial ring in $2 m n$ variables; also let $R_{x}=\mathbb{F}\left[V_{x}\right]$ and $R_{y}=\mathbb{F}\left[V_{y}\right]$ be the corresponding polynomial rings in $m n$ variables. By the support of a monomial $F$ in $R$, denoted $\operatorname{supp}(F)$, we mean the subset of $V$ consisting of the variables appearing in $F$. Clearly a monomial $F$ in $R$ can be uniquely written as

$$
F=F_{x} F_{y}, \quad \text { where } F_{x}, F_{y} \text { are monomials with } F_{x} \in R_{x} \text { and } F_{y} \in R_{y},
$$

and moreover $F$ is squarefree if and only if both $F_{x}$ and $F_{y}$ are squarefree. Note that squarefree monomials can be identified with their supports, and in particular, faces of a simplicial complex $\Delta$ with vertex set $V$ can be viewed as squarefree monomials in $R$. With this in view, we may not distinguish between a squarefree monomial and its support, and we may sometimes write $x_{i, j} \in G$ rather than $x_{i, j} \mid G$ when $G$ is a squarefree monomial in $R$ and $x_{i, j}$ is a variable appearing in it. A monomial $G$ in $R_{x}$ will be called a lattice path monomial in $R_{x}$ if there is a positive integer $t$ and variables $x_{i_{1}, j_{1}}, \ldots, x_{i_{t}, j_{t}}$ in $V_{x}$ such that

$$
G=\prod_{s=1}^{t} x_{i_{s}, j_{s}} \quad \text { with }\left(i_{s}-i_{s-1}, j_{s}-j_{s-1}\right)=(1,0) \text { or }(0,1) \text { for } 1<s \leq t .
$$

In this case $G$ is called a lattice path monomial from $x_{i_{1}, j_{1}}$ to $x_{i_{t}, j_{t}}$, and we will refer to $x_{i_{1}, j_{1}}$ as the leader of $G$ and denote it by $\mu(G)$. Note that $\mu(G)=x_{i_{1}, j_{1}}$ depends only on $G$ (and not on the given ordering of the variables appearing in it) since $\left(i_{1}, j_{1}\right)$ is lexicographically the least among the pairs $(i, j)$ for which $x_{i, j} \in \operatorname{supp}(G)$. A variable $x_{i_{s}, j_{s}}$ in $\operatorname{supp}(G)$ will be called an ES-turn of $G$ if $1<s<t, i_{s}=i_{s-1}$, and $j_{s}=j_{s+1}$. Analogously a variable $x_{i_{s}, j_{s}}$ in $\operatorname{supp}(G)$ will be called a $S E$-turn of $G$ if $1<s<t, j_{s}=j_{s-1}$, and $i_{s}=i_{s+1}$. Moreover we will call a variable $x_{i_{s}}, j_{s}$ in $\operatorname{supp}(G)$ the midpoint of a segment in $G$ if $1<s<t$ and either $i_{s-1}=i_{s}=i_{s+1}$ (horizontal segment) or $j_{s-1}=j_{s}=j_{s+1}$ (vertical segment). It may be noted that a variable $x_{i_{s}, j_{s}}$ with $1<s<t$ is either an ES-turn or a SE-turn or the midpoint of a segment in $G$.

Evidently lattice path monomials in $R_{x}$ correspond to lattice paths in the sense of Section 2.3 if we turn the $m \times n$ rectangular matrix $\left(x_{i, j}\right)$ left by $90^{\circ}$ and identify the variable $x_{i, j}$ with the lattice point $(i, j)$. In this way leaders correspond to initial 

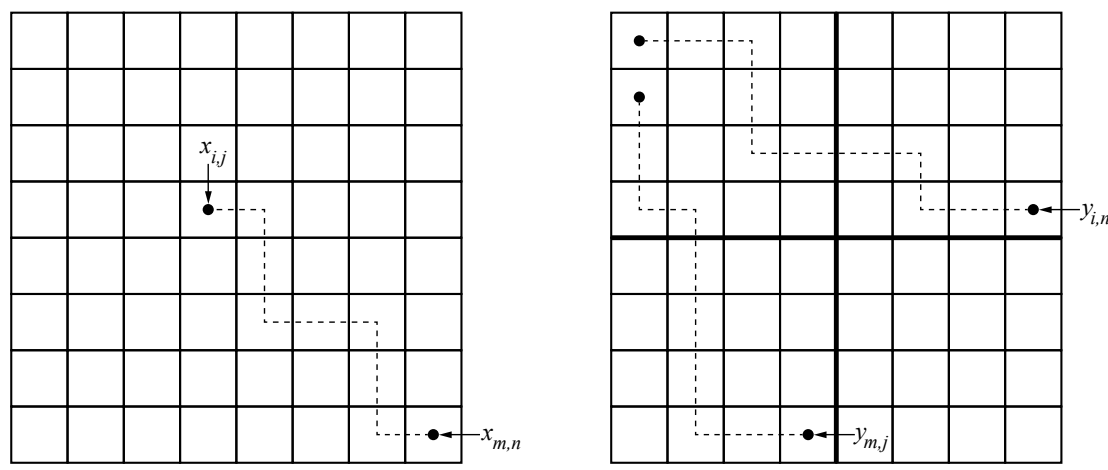

Figure 2. Lattice path monomials $F_{x}$ and $F_{y}=F_{y}^{\mathrm{U}} F_{y}^{\mathrm{L}}$ in Proposition 5.

points while ES turns correspond to NE turns. Lattice path monomials in $R_{y}$ together with their leaders, ES turns, SE turns, and midpoints of segments are similarly defined (and similarly identified with lattice paths in the sense of Section 2.3).

We have noted in the introduction that a Gröbner basis (with respect to reverse lexicographic order on monomials with the $2 m n$ variables arranged suitably) of the ideal $\mathscr{I}$ of the variety $\mathscr{L}_{2,2}^{m, n}$ of first-order jets over $\mathscr{E}_{2}^{m, n}$, as well as of the ideal $\mathscr{I}_{0}$ of the principal component $Z_{0}$ of $\mathscr{E}_{2,2}^{m, n}$, was determined in [Košir and Sethuraman 2005b]. As a consequence, one can write down the generators of the leading term ideal of $\mathscr{I}_{0}$ (see [Jonov 2011, Proposition 1.1]), say LT $\left(\Phi_{0}\right)$, and deduce that $R / \mathrm{LT}\left(\mathscr{I}_{0}\right)$ is the Stanley-Reisner ring of a simplicial complex $\Delta_{0}$ with $V$ as its set of vertices. A precise description of the facets of $\Delta_{0}$ was given by Jonov [2011, $\S 2]$, and we recall it below.

Proposition 5. A squarefree monomial $F$, decomposed as in (15) above, is a facet of $\Delta_{0}$ if and only if there is a unique $(i, j) \in \mathbb{Z}^{2}$, with $1 \leq i \leq m, 1 \leq j \leq n$, such that $(i, j) \neq(m, n)$ and $F_{x}$ is a lattice path monomial from $x_{i, j}$ to $x_{m, n}$, whereas $F_{y}=F_{y}^{\mathrm{U}} F_{y}^{\mathrm{L}}$, where $F_{y}^{\mathrm{U}}$ is a lattice path monomial from $y_{1,1}$ to $y_{i, n}, F_{y}^{\mathrm{L}}$ is a lattice path monomial from $y_{2,1}$ to $y_{m, j}$, and the supports of $F_{y}^{\mathrm{U}}$ and $F_{y}^{\mathrm{L}}$ are disjoint.

The lattice path monomials $F_{x}$ and $F_{y}=F_{y}^{U} F_{y}^{\mathrm{L}}$ are illustrated in Figure 2 by the corresponding "paths" in rectangular matrices.

Using Proposition 5 together with the first identity in (12) and part (i) of Proposition 3, Jonov showed that the simplicial complex $\Delta_{0}$ is pure (i.e., all its facets have the same dimension) and deduced the dimension and the formula stated in the introduction for the multiplicity of the coordinate ring $R / \mathscr{I}_{0}$ of $Z_{0}$.

Corollary 6. The (Krull) dimension of $R / \mathscr{I}_{0}$ is $2(m+n-1)$ and the multiplicity of $R / I_{0}$ is given by (3).

Now here is the pretty result about the multiplicity that was alluded to in the introduction, namely, that the formula (3) admits a remarkable simplification. 
Theorem 7. The multiplicity of $R / \mathscr{I}_{0}$ is given by

$$
e\left(R / \mathscr{I}_{0}\right)=\left(\begin{array}{c}
m+n-2 \\
m-1
\end{array}\right)^{2}
$$

Proof. For $1 \leq i \leq m$ and $1 \leq j \leq n$, let $\Delta_{i, j}$ denote the $2 \times 2$ determinant in (3). Observe that if $(i, j)=(m, n)$, then $\Delta_{i, j}=0$. Thus, by expanding this determinant and rearranging the summands, we can write

$$
e\left(R / \mathscr{I}_{0}\right)=\sum_{i=1}^{m}\left(\begin{array}{c}
i+n-2 \\
i-1
\end{array}\right) \sum_{j=1}^{n} S_{i, j}-\sum_{i=1}^{m}\left(\begin{array}{c}
i+n-3 \\
i-2
\end{array}\right) \sum_{j=1}^{n} T_{i, j},
$$

where, for $1 \leq i \leq m$ and $1 \leq j \leq n$, we have put

$$
S_{i, j}=\left(\begin{array}{c}
m+n-i-j \\
m-i
\end{array}\right)\left(\begin{array}{c}
m+j-3 \\
m-2
\end{array}\right) \text { and } T_{i, j}=\left(\begin{array}{c}
m+n-i-j \\
m-i
\end{array}\right)\left(\begin{array}{c}
m+j-2 \\
m-1
\end{array}\right) .
$$

Rewriting $S_{i, j}$ using (5) and then noting that the resulting product is zero if $j<1$ or $j>n$, thanks to (4), we see from Equation (9) in Lemma 2 that

$$
\sum_{j=1}^{n} S_{i, j}=\sum_{j}\left(\begin{array}{c}
m+n-i-j \\
n-j
\end{array}\right)\left(\begin{array}{c}
m+j-3 \\
j-1
\end{array}\right)=\left(\begin{array}{c}
2 m+n-i-2 \\
n-1
\end{array}\right),
$$

for each $i=1, \ldots, m$. In a similar manner,

$$
\sum_{j=1}^{n} T_{i, j}=\sum_{j}\left(\begin{array}{c}
m+n-i-j \\
n-j
\end{array}\right)\left(\begin{array}{c}
m+j-2 \\
j-1
\end{array}\right)=\left(\begin{array}{c}
2 m+n-i-1 \\
n-1
\end{array}\right),
$$

for each $i=1, \ldots, m$. It follows that $e\left(R / \mathscr{I}_{0}\right)$ is given by the telescoping sum

$$
e\left(R / \mathscr{I}_{0}\right)=\sum_{i=1}^{m}\left(a_{i}-a_{i-1}\right), \quad \text { where } a_{i}:=\left(\begin{array}{c}
i+n-2 \\
i-1
\end{array}\right)\left(\begin{array}{c}
2 m+n-i-2 \\
n-1
\end{array}\right),
$$

for $0 \leq i \leq m$. Since $a_{0}=0$ and $a_{m}=\left(\begin{array}{c}m+n-2 \\ m-1\end{array}\right)^{2}$, we obtain the desired result.

It may be noted that in view of (1) and (17), the multiplicity of the principal component $Z_{0}$ is precisely the square of the multiplicity of the base variety $\mathscr{L}_{2}^{m, n}$.

\section{Hilbert series}

Let us begin by recalling that a shelling of a pure simplicial complex $\Delta$ is a linear ordering $F_{1}, \ldots, F_{e}$ of its facets such that for all positive integers $i, j$, with $j<i \leq e$, there exist some $v \in F_{i} \backslash F_{j}$ and some positive integer $k<i$ such that $F_{i} \backslash F_{k}=\{v\}$. Given such a shelling and any $t \in\{1, \ldots, e\}$, we let

$$
c\left(F_{t}\right)=\left\{v \in F_{t}: \text { there exists } s<t \text { such that } F_{t} \backslash F_{s}=\{v\}\right\} .
$$


Elements of $c\left(F_{t}\right)$ will be referred to as the corners of $F_{t}$. It may be noted that $c\left(F_{t}\right)$ is nonempty if and only if $t>1$. Recall also that a simplicial complex $\Delta$ is said to be shellable if it is pure and it has a shelling. The following result is well known (see [Bruns and Conca 2003, Theorem 6.3]).

Proposition 8. Let $\Delta$ be a shellable simplicial complex and let $R_{\Delta}$ denote its Stanley-Reisner ring. Then:

(i) $R_{\Delta}$ is Cohen-Macaulay and its (Krull) dimension $\operatorname{dim} R_{\Delta}$ is $1+\operatorname{dim} \Delta$.

(ii) Suppose $d=\operatorname{dim} R_{\Delta}$ and $F_{1}, \ldots, F_{e}$ is a shelling of $\Delta$. Then the Hilbert series of $R_{\Delta}$ is given by

$$
\frac{\sum_{j \geq 0} h_{j} z^{j}}{(1-z)^{d}}, \quad \text { where } h_{j}=\left|\left\{t \in\{1, \ldots, e\}:\left|c\left(F_{t}\right)\right|=j\right\}\right| \text { for } j \geq 0 .
$$

Jonov [2011] showed that the simplicial complex $\Delta_{0}$ mentioned in the previous section is shellable and concluded using part (i) of Proposition 8 that the coordinate ring of $R / I_{0}$ of the principal component $Z_{0}$ of $\mathscr{L}_{2,2}^{m, n}$ is Cohen-Macaulay. We shall now proceed to use part (ii) of Proposition 8 to determine the Hilbert series of $R / \mathscr{I}_{0}$. We will use the notation and terminology introduced at the beginning of Section 3. Further we introduce the following "antilexicographic" linear order on $V_{x}$, that is, on the $x$-variables. For any $x_{a, b}, x_{c, d} \in V_{x}$, define

$$
x_{a, b} \prec x_{c, d} \Longleftrightarrow \text { either } a>c \quad \text { or } \quad a=c \text { and } b>d .
$$

Given a lattice path monomial $G$ as in (16), the spread of $G$, denoted $\operatorname{sp}(G)$, is the set of variables that are on or below the corresponding lattice path; more precisely,

$$
\operatorname{sp}(G)=\left\{x_{a, b}: i_{s} \leq a \leq m \text { and } 1 \leq b \leq j_{s} \text { for some } s=1, \ldots, t\right\} .
$$

The notion of spread is defined for lattice path monomials in $R_{y}$ in exactly the same manner. It may be observed that if $G, H$ are lattice path monomials (both in $R_{x}$ or both in $R_{y}$ ), then the condition $\operatorname{sp}(G) \subseteq \operatorname{sp}(H)$ means, roughly speaking, that $H$ is to the right of $G$; moreover, if $\mu(G)=\mu(H)$ and $\operatorname{sp}(G)=\operatorname{sp}(H)$, then we must have $G=H$.

Notice that the lattice path monomials $F_{y}^{U}$ and $F_{y}^{\mathrm{L}}$ of Proposition 5 have the property that $\operatorname{sp}\left(F_{y}^{\mathrm{L}}\right) \subseteq \operatorname{sp}\left(F_{y}^{\mathrm{U}}\right)$.

Following [Jonov 2011], we now define a partial order on the facets of $\Delta_{0}$.

Definition 9. For any facets $P, Q$ of $\Delta_{0}$ with decompositions $P=P_{x} P_{y}^{\mathrm{U}} P_{y}^{\mathrm{L}}$ and $Q=Q_{x} Q_{y}^{\mathrm{U}} Q_{y}^{\mathrm{L}}$ as in Proposition 5, define $P<Q$ if one of the following four conditions hold: (i) $\mu\left(P_{x}\right) \prec \mu\left(Q_{x}\right)$, (ii) $\mu\left(P_{x}\right)=\mu\left(Q_{x}\right)$ and $\operatorname{sp}\left(P_{x}\right) \subsetneq \operatorname{sp}\left(Q_{x}\right)$, (iii) $P_{x}=Q_{x}$ and $\operatorname{sp}\left(P_{y}^{\mathrm{U}}\right) \subsetneq \operatorname{sp}\left(Q_{y}^{\mathrm{U}}\right)$, (iv) $P_{x}=Q_{x}, P_{y}^{\mathrm{U}}=Q_{y}^{\mathrm{U}}$ and $\operatorname{sp}\left(P_{y}^{\mathrm{L}}\right) \subsetneq \operatorname{sp}\left(Q_{y}^{\mathrm{L}}\right)$.

The next result is a consequence of [Jonov 2011, Theorem 3.2] and its proof. 
Proposition 10. The relation < in Definition 9 defines a partial order and any extension of it to a total order on the facets of $\Delta_{0}$ gives a shelling of $\Delta_{0}$.

The terminology of ES turns can be extended from lattice path monomials to facets of $\Delta_{0}$ as follows. For any facet $F$ of $\Delta_{0}$ having a decomposition $F=F_{x} F_{y}^{\mathrm{U}} F_{y}^{1}$ as in Proposition 5, by an ES-turn of $F$ we shall mean an ES-turn of either $F_{x}$ or $F_{y}^{\mathrm{L}}$ or $F_{y}^{U}$. It turns out that the corners of a facet of $\Delta_{0}$ are essentially its ES turns or the leader of its $x$-component. There are, however, some subtleties involved and a precise relation is given below.

Lemma 11. Let $F$ be a facet of $\Delta_{0}$ and $F=F_{x} F_{y}^{\mathrm{U}} F_{y}^{\mathrm{L}}$ be its decomposition as in Proposition 5. Also let $v \in V$ be a vertex of $\Delta_{0}$. Then:

(i) If $v \in c(F)$, then either $v=\mu\left(F_{x}\right)$ or $v$ is an ES-turn of $F$. In particular, $x_{m, n} \notin c(F)$ and $y_{m, n} \notin c(F)$.

(ii) If $\mu\left(F_{x}\right)=x_{i, j}$, with $(i, j) \neq(m, n-1)$, then $\mu\left(F_{x}\right) \in c(F)$. Moreover $x_{m, n-1} \notin c(F)$.

(iii) If $v$ is an ES-turn of $F_{x}$, then $v \in c(F)$.

(iv) If $v$ is an ES-turn of $F_{y}^{\mathrm{U}}$ or of $F_{y}^{\mathrm{L}}$, then $v \in c(F)$, except when $v$ is an ES-turn of $F_{y}^{U}$ such that $v=y_{1,2}$ or when $v$ is an ES-turn of $F_{y}^{U}$ such that $v=y_{m-1, j+1}$ and $\mu\left(F_{x}\right)=x_{m, j}$ for some $j<n$.

Proof. (i) Let $P=P_{x} P_{y}^{\mathrm{U}} P_{y}^{\mathrm{L}}$ be a facet of $\Delta_{0}$ such that $F \backslash P=\{v\}$ and $F>P$. The latter implies that one of the four possibilities in Definition 9 must arise. First suppose $\mu\left(P_{x}\right) \prec \mu\left(F_{x}\right)$. Then $\mu\left(F_{x}\right)$ is a vertex of $F$ that is smaller than $\mu\left(P_{x}\right)$ in the standard lexicographic order, and hence $\mu\left(F_{x}\right) \notin P_{x}$; consequently $v=\mu\left(F_{x}\right)$, and we are done. Now suppose $\mu\left(P_{x}\right)=\mu\left(F_{x}\right)$ and $\operatorname{sp}\left(P_{x}\right) \subsetneq \operatorname{sp}\left(F_{x}\right)$. Then $P_{x} \neq F_{x}$ and hence $F_{x} \backslash P_{x}=\{v\}$. Note that since $\mu\left(F_{x}\right)$ and $x_{m, n}$ are in $P_{x}$, the vertex $v$ is an ES-turn, SE-turn, or the midpoint of a segment of $F_{x}$. In case it is the midpoint of a segment of $F_{x}$, the other two vertices in that segment must be in $P_{x}$, and since $P_{x}$ is a lattice path monomial, we see that $v \in P_{x}$, which is a contradiction. Also if $v=x_{k, l}$ (say) is a SE-turn of $F_{x}$, then $x_{k-1, l}$ and $x_{k, l+1}$ must be in $F_{x}$ and hence in $P_{x}$. But then $P_{x}$ must contain $x_{k-1, l+1}$, which is a contradiction since $x_{k-1, l+1} \notin \operatorname{sp}\left(F_{x}\right)$. It follows that $v$ is an ES-turn of $F_{x}$. Next suppose $P_{x}=F_{x}$ and $\operatorname{sp}\left(P_{y}^{\mathrm{U}}\right) \subsetneq \operatorname{sp}\left(F_{y}^{\mathrm{U}}\right)$. Then $F_{y}^{\mathrm{U}} \backslash P_{y}^{\mathrm{U}}=\{v\}$. Since $\mu\left(P_{x}\right)=\mu\left(F_{x}\right)$, in view of Proposition 5, we see that the initial and the terminal variables of $P_{y}^{\mathrm{U}}$ and $F_{y}^{\mathrm{U}}$ coincide, and so $v$ is neither of these. Arguing as in the preceding case, we can rule out the possibilities that $v$ is a SE-turn or the midpoint of a segment of $F_{y}^{U}$. Hence $v$ is an ES-turn of $F_{y}^{U}$. In a similar manner, we see that if $P_{x}=F_{x}, P_{y}^{U}=F_{y}^{U}$ and $\operatorname{sp}\left(P_{y}^{\mathrm{L}}\right) \subsetneq \operatorname{sp}\left(F_{y}^{\mathrm{L}}\right)$, then $v$ is a ES-turn of $F_{y}^{\mathrm{L}}$. Thus (i) is proved.

(ii) Let $\mu\left(F_{x}\right)=x_{i, j}$ with $(i, j) \neq(m, n-1)$. Then either $x_{i, j+1} \in F_{x}$ or $x_{i+1, j} \in F_{x}$. First suppose $x_{i, j+1} \in F_{x}$. We define a new facet $P$ as follows. Let $P_{x}=F_{x} \backslash\left\{x_{i, j}\right\}$ 
and $P_{y}^{\mathrm{L}}=F_{y}^{\mathrm{L}} \cup\left\{y_{m, j+1}\right\}$. To define $P_{y}^{\mathrm{U}}$, we take $P_{y}^{\mathrm{U}}=F_{y}^{\mathrm{U}}$ in the case $y_{m, j+1} \notin F_{y}^{\mathrm{U}}$. If $y_{m, j+1} \in F_{y}^{\mathrm{U}}$, then this must mean that $i=m$, and hence $j<n-1$. We therefore define $P_{y}^{\mathrm{U}}=\left(F_{y}^{\mathrm{U}} \backslash\left\{y_{m, j+1}\right\}\right) \cup\left\{y_{m-1, j+2}\right\}$. Observe that $P=P_{x} P_{y}^{\mathrm{U}} P_{y}^{\mathrm{L}}$ is a facet of $\Delta_{0}$ and since $\mu\left(P_{x}\right) \prec \mu\left(F_{x}\right)$, we have $P<F$. It follows that $\mu\left(F_{x}\right) \in c(F)$. Next suppose $x_{i+1, j} \in F_{x}$. We first assume that $(i, j) \neq(m-1, n)$. Now define a new facet $P$ as follows. First we let $P_{x}=F_{x} \backslash\left\{x_{i, j}\right\}$. If $y_{i+1, n} \notin F_{y}^{\mathrm{L}}$, then we let $P_{y}^{\mathrm{U}}=F_{y}^{\mathrm{U}} \cup\left\{y_{i+1, n}\right\}$ and $P_{y}^{\mathrm{L}}=F_{y}^{\mathrm{L}}$. If $y_{i+1, n} \in F_{y}^{\mathrm{L}}$, then $j$ must equal $n$. If now $i \leq m-2$, then we let $P_{y}^{\mathrm{L}}=\left(F_{y}^{\mathrm{L}} \backslash\left\{y_{i+1, n}\right\}\right) \cup\left\{y_{i+2, n-1}\right\}$. We are left with the special case $i=m-1, j=n$. Here we let $P_{x}=\left\{x_{m, n-1}, x_{m, n}\right\}, P_{y}^{\cup}=F_{y}^{\cup} \cup\left\{y_{m, n}\right\}$, and $P_{y}^{\mathrm{L}}=F_{y}^{\mathrm{L}} \backslash\left\{y_{m, n}\right\}$. In all three cases, it is easy to verify that $P=P_{x} P_{y}^{\mathrm{U}} P_{y}^{\mathrm{L}}$ is a facet of $\Delta_{0}$ such that $F \backslash P=\left\{x_{i, j}\right\}$ and $P<F$. Consequently $\mu\left(F_{x}\right) \in c(F)$. Finally we show that $x_{m, n-1} \notin c(F)$. Assume, on the contrary, that there is a facet $P$ of $\Delta_{0}$ such that $F \backslash P=\left\{x_{m, n-1}\right\}$. By (i) above, $\mu(F)=x_{m, n-1}$ because there can be no ES-turn at $x_{m, n-1}$. In view of Proposition 5, $P$ must contain at least one variable other than $x_{m, n}$, and since $x_{m, n-1} \notin P$, it follows that $x_{m-1, n} \in P$. This forces $\mu\left(F_{x}\right) \prec \mu\left(P_{x}\right)$, which violates the fact that $P<F$. Thus (ii) is proved.

(iii) Let $v=x_{k, l}$ be an ES-turn of $F_{x}$. Define $P_{x}=F_{x} \backslash\left\{x_{k, l}\right\} \cup\left\{x_{k+1, l-1}\right\}$ and $P_{y}=F_{y}$. It is clear that $P=P_{x} P_{y}$ is a facet of $\Delta_{0}$ such that $P<F$ and $F \backslash P=\{v\}$. This proves (iii).

(iv) First suppose $v=y_{k, l}$ is an ES-turn of $F_{y}^{\mathrm{L}}$. Then $k<m$ and $l>1$. Define $P_{x}=F_{x}, P_{y}^{\mathrm{U}}=F_{y}^{\mathrm{U}}$, and $P_{y}^{\mathrm{L}}=F_{y}^{\mathrm{L}} \backslash\left\{y_{k, l}\right\} \cup\left\{y_{k+1, l-1}\right\}$. It is easy to see that $P=P_{x} P_{y}^{\mathrm{U}} P_{y}^{\mathrm{L}}$ is facet of $\Delta_{0}$ such that $P<F$ and $F \backslash P=\{v\}$. Next suppose $v=y_{k, l}$ is an ES-turn of $F_{y}^{\mathrm{U}}$. Then once again $k<m$ and $l>1$. In case $y_{k+1, l-1}$ is not in $F_{y}^{\mathrm{L}}$, we define $P_{x}=F_{x}, P_{y}^{\mathrm{L}}=F_{y}^{\mathrm{L}}$, and $P_{y}^{\mathrm{U}}=F_{y}^{\mathrm{U}} \backslash\left\{y_{k, l}\right\} \cup\left\{y_{k+1, l-1}\right\}$, whereas in case $y_{k+1, l-1}$ is in $F_{y}^{\mathrm{L}}$ and also $k<m-1$ and $l>2$, we define $P_{x}=F_{x}$, $P_{y}^{\mathrm{U}}=F_{y}^{\mathrm{U}} \backslash\left\{y_{k, l}\right\} \cup\left\{y_{k+1, l-1}\right\}$, and $P_{y}^{\mathrm{L}}=F_{y}^{\mathrm{L}} \backslash\left\{y_{k+1, l-1}\right\} \cup\left\{y_{k+2, l-2}\right\}$. We verify that in both the cases, $P=P_{x} P_{y}^{\mathrm{U}} P_{y}^{\mathrm{L}}$ is a facet of $\Delta_{0}$ such that $P<F$ and $F \backslash P=\{v\}$.

When $l=2$, it is easy to see that $v=y_{k, 2}$ can be an ES-turn of $F_{y}^{\mathrm{U}}$ only when $k=1$ lest $F_{y}^{\mathrm{U}}$ and $F_{y}^{\mathrm{L}}$ intersect at $y_{k, 1}$. We now show that $y_{1,2}$ is not a corner of $F$. Suppose that $P=P_{x} P_{y}^{\mathrm{U}} P_{y}^{\mathrm{L}}$ is a facet of $\Delta_{0}$ such that $F \backslash P=\{v\}, v=y_{1,2}$ and $F>P$. By Proposition 5, $P_{y}^{U}$ must start at $y_{1,1}$ and $P_{y}^{\mathrm{L}}$ must start at $y_{2,1}$. For $P_{y}^{\mathrm{U}}$ to avoid $v=y_{1,2}$, it must be the case that $P_{y}^{\mathrm{U}}$ contains $y_{2,1}$. But this contradicts the fact that $P_{y}^{\mathrm{U}}$ and $P_{y}^{\mathrm{L}}$ do not intersect.

We are left with the situation where $k=m-1$ and $v=y_{k, l}$ is an ES-turn of $F_{y}^{U}$ and moreover $y_{m, l-1} \in F_{y}^{\mathrm{L}}$. Now since $F_{y}^{\mathrm{U}}$ has an ES-turn at $y_{m-1, l}$, we see that $l>1$ and both $y_{m-1, l-1}$ and $y_{m, l}$ are in $F_{y}^{\mathrm{U}}$. In particular, $y_{m, l} \notin F_{y}^{\mathrm{L}}$ and since $y_{m, l-1} \in F_{y}^{\mathrm{L}}$, in view of Proposition 5, it follows that $F_{y}^{\mathrm{L}}$ ends at $y_{m, l-1}$, while $F_{y}^{\mathrm{U}}$ ends at $y_{m, n}$ and also that $\mu\left(F_{x}\right)=x_{m, l-1}$. Now if there were a facet $P=P_{x} P_{y}^{\mathrm{U}} P_{y}^{\mathrm{L}}$ of $\Delta_{0}$ such that $F \backslash P=\{v\}$ and $F>P$, then $P_{x}=F_{x}$ and $P_{y}^{\mathrm{L}}=F_{y}^{\mathrm{L}}$, whereas 
$F_{y}^{\mathrm{U}} \backslash P_{y}^{\mathrm{U}}=\left\{y_{m-1, l}\right\}$. But then $P_{y}^{\mathrm{U}}$ is a lattice path monomial that contains both $y_{m-1, l-1}$ and $y_{m, l}$ and does not contain $y_{m-1, l}$; so it must contain $y_{m, l-1}$. This is a contradiction since $y_{m, l-1} \in F_{y}^{\mathrm{L}}=P_{y}^{\mathrm{L}}$ and the monomials $P_{y}^{\mathrm{U}}$ and $P_{y}^{\mathrm{L}}$ have no variable in common. This completes the proof.

For any integers $i, j, k$ with $k \geq 0,1 \leq i \leq m$ and $1 \leq j \leq n$, we define $C_{i, j}^{k}$ to be the number of facets $F=F_{x} F_{y}$ of $\Delta_{0}$ such that $\mu\left(F_{x}\right)=x_{i, j}$ and $F$ has exactly $k$ ES turns that are in $c(F)$. We state a useful consequence of Lemma 11:

Corollary 12. The Hilbert series of the coordinate ring $R / \mathscr{I}_{0}$ of the principal component $Z_{0}$ of $\mathscr{E}_{2,2}^{m, n}$ is given by

$$
\frac{\sum_{k \geq 0} h_{k} z^{k}}{(1-z)^{2(m+n-1)}}
$$

where $h_{0}=1$, and for $k \geq 1$,

$$
h_{k}=C_{m, n-1}^{k}+\sum_{\substack{i, j) \neq(m, n-1) \\ i, j) \neq(m, n)}} C_{i, j}^{k-1},
$$

where the last sum is over all pairs $(i, j)$ of integers satisfying $1 \leq i \leq m$ and $1 \leq j \leq n$, with $(i, j) \neq(m, n-1)$ and $(i, j) \neq(m, n)$.

Proof. It is well-known that the (Krull) dimension as well as the Hilbert series of $R / \Phi_{0}$ coincides with that of $R / \mathrm{LT}\left(\mathscr{I}_{0}\right)$ (see, e.g., [Bruns and Conca 2003, §3]), where $\operatorname{LT}\left(\mathscr{I}_{0}\right)$ denotes the leading term ideal of $\mathscr{I}_{0}$ as in [Košir and Sethuraman 2005b] and [Jonov 2011, Proposition 1.1]. Now $\Delta_{0}$ is precisely the simplicial complex such that $R / \mathrm{LT}\left(\mathscr{I}_{0}\right)$ is the Stanley-Reisner ring of $\Delta_{0}$. Thus it follows from Corollary 6 and part (ii) of Proposition 8 that the Hilbert series of $R / \mathscr{I}_{0}$ is given by (18), where $h_{0}=1$, and for $k \geq 1$,

$$
h_{k}=\mid\left\{F: F \text { a facet of } \Delta_{0} \text { with }|c(F)|=k\right\} \mid .
$$

Partitioning the facets $F=F_{x} F_{y}$ in the above set in accordance with the values of $\mu\left(F_{x}\right)$ and noting from Proposition 5 that $\mu\left(F_{x}\right) \neq(m, n)$, and then applying Lemma 11, we obtain the desired result.

We have seen in Section 3 that lattice path monomials can be related to lattice paths in the sense of Section 2.3 if we rotate to the left by $90^{\circ}$ and identify the variable $x_{i, j}$ with the point $(i, j)$ of $\mathbb{Z}^{2}$. Also recall that for any $\left(a, a^{\prime}\right),\left(e, e^{\prime}\right) \in \mathbb{Z}^{2}$ and $s \in \mathbb{Z}$, we denote by $\mathscr{P}_{s}\left(\left(a, a^{\prime}\right) \rightarrow\left(e, e^{\prime}\right)\right)$ the set of lattice paths from $\left(a, a^{\prime}\right)$ to $\left(e, e^{\prime}\right)$ with $s$ NE turns. Likewise if $\left(a_{i}, a_{i}^{\prime}\right),\left(e_{i}, e_{i}^{\prime}\right) \in \mathbb{Z}^{2}$ for $i=1,2$ and $s \in \mathbb{Z}$, then by $\mathscr{P}_{s}\left(\left(a_{1}, a_{1}^{\prime}\right) \rightarrow\left(e_{1}, e_{1}^{\prime}\right),\left(a_{2}, a_{2}^{\prime}\right) \rightarrow\left(e_{2}, e_{2}^{\prime}\right)\right)$ we denote the set of pairs $\left(L_{1}, L_{2}\right)$ of nonintersecting lattice paths such that $L_{i}$ is from $\left(a_{i}, a_{i}^{\prime}\right)$ to $\left(e_{i}, e_{i}^{\prime}\right)$ for $i=1,2$, 

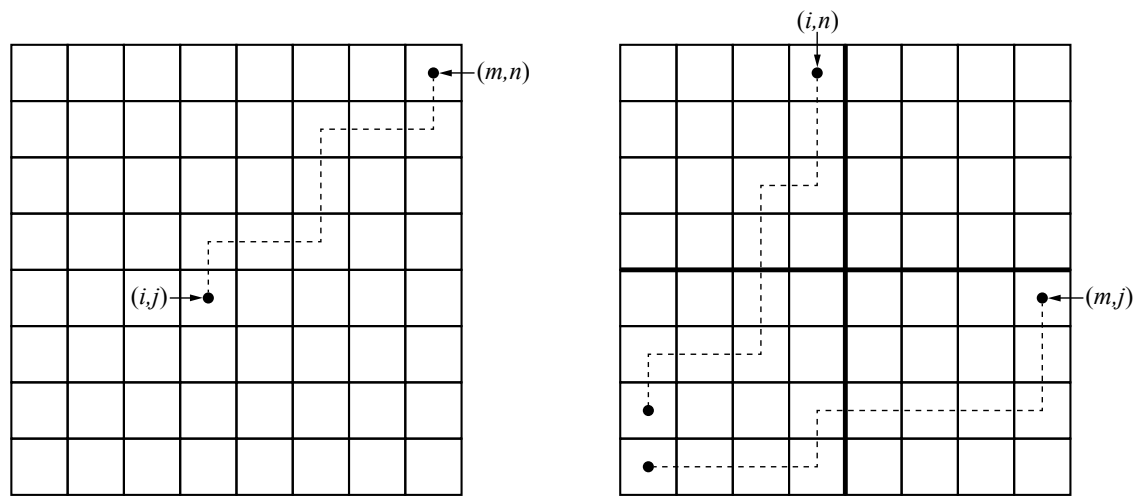

Figure 3. Lattice paths $L$ and $\left(L_{1}, L_{2}\right)$ corresponding to $F_{x}$ and $\left(F_{y}^{U}, F_{y}^{\mathrm{L}}\right)$.

and the paths $L_{1}$ and $L_{2}$ together have exactly $s$ NE turns. Evidently these sets are empty (and hence of cardinality 0 ) when $s<0$.

Lemma 13. Let $s, i, j \in \mathbb{Z}$ with $s \geq 0,1 \leq i \leq m$ and $1 \leq j \leq n$.

(i) If $i \neq m$, then

$$
C_{i, j}^{s}=\sum_{s_{1}+s_{2}=s}\left|\mathscr{P}_{s_{1}}((i, j) \rightarrow(m, n))\right|\left|\mathscr{P}_{s_{2}}((1,2) \rightarrow(i, n),(1,1) \rightarrow(m, j))\right|,
$$

where the sum is over pairs $\left(s_{1}, s_{2}\right)$ of nonnegative integers with $s_{1}+s_{2}=s$.

(ii) If $1<j<n-1$, then

$$
\begin{aligned}
C_{m, j}^{s}=\sum_{p=1}^{m-1} & \sum_{q=j+1}^{n-1}\left|\mathscr{P}_{s-1}((1,2) \rightarrow(p, q),(1,1) \rightarrow(m, j))\right| \\
& +\sum_{p=1}^{m-2}\left|\mathscr{P}_{s-1}((1,2) \rightarrow(p, j),(1,1) \rightarrow(m, j))\right| \\
& +\left|\mathscr{P}_{s}((1,2) \rightarrow(m-1, j),(1,1) \rightarrow(m, j))\right| .
\end{aligned}
$$

(iii) $C_{m, 1}^{s}=\left(\begin{array}{c}n-2 \\ s\end{array}\right)\left(\begin{array}{c}m-1 \\ s\end{array}\right)$ and

$$
\begin{aligned}
C_{m, n-1}^{s}=\sum_{p=1}^{m-2}\left|\mathscr{P}_{s-1}((1,2) \rightarrow(p, n-1),(1,1) \rightarrow(m, n-1))\right| \\
+\left|\mathscr{P}_{s}((1,2) \rightarrow(m-1, n-1),(1,1) \rightarrow(m, n-1))\right| .
\end{aligned}
$$

Proof. Let $i, j \in \mathbb{Z}$ with $1 \leq i \leq m, 1 \leq j \leq n$, and $(i, j) \neq(m, n)$. By a $90^{\circ}$ rotation to the left, we see from Proposition 5 that the facets $F=F_{x} F_{y}$ of $\Delta_{0}$ with $\mu\left(F_{x}\right)=x_{i, j}$ are in one-to-one correspondence with the triples $\left(L, L_{1}^{*}, L_{2}^{*}\right)$ 
of lattice paths, where $L$ is from $(i, j)$ to $(m, n)$, while $L_{1}^{*}$ is from $(1,1)$ to $(i, n)$ and $L_{2}^{*}$ is from $(2,1)$ to $(m, j)$, and moreover $L_{1}^{*}, L_{2}^{*}$ are nonintersecting. We will now modify $L_{1}^{*}, L_{2}^{*}$ slightly keeping in mind the hypothesis in Corollary 4 . To this end, first note that $(1,2) \in L_{1}^{*}$ since $2<m \leq n$. Thus if we let $L_{1}:=L_{1}^{*} \backslash\{(1,1)\}$ and $L_{2}:=L_{2}^{*} \cup\{(1,1)\}$, then $\left(L_{1}^{*}, L_{2}^{*}\right)$ and $\left(L_{1}, L_{2}\right)$ are pairs of nonintersecting lattice paths that determine each other and have exactly the same NE turns, except that if $L_{1}^{*}$ had a NE turn at $(1,2)$, then $L_{1}$ will not have a NE turn at $(1,2)$. Note though that, by Lemma 11 (iv), $y_{1,2}$ is not a corner of any facet, and this switch will therefore not affect the count of corners. Consequently the facets $F=F_{x} F_{y}$ of $\Delta_{0}$ with $\mu\left(F_{x}\right)=x_{i, j}$ are in one-to-one correspondence with

$$
\mathscr{P}((i, j) \rightarrow(m, n)) \times \mathscr{P}((1,2) \rightarrow(i, n),(1,1) \rightarrow(m, j)) .
$$

The lattice paths $L$ and $\left(L_{1}, L_{2}\right)$ corresponding to the components $F_{x}$ and $\left(F_{y}^{\mathrm{U}}, F_{y}^{\mathrm{L}}\right)$ of the facet $F=F_{x} F_{y}$ are illustrated in Figure 3; these may be compared with Figure 2 that depicts the lattice path monomials $F_{x}$ and $F_{y}=F_{y}^{\mathrm{U}} F_{y}^{\mathrm{L}}$.

(i) Suppose $i \neq m$. Then, from Lemma 11, we see that, for every facet $F=F_{x} F_{y}$ of $\Delta_{0}$ with $\mu\left(F_{x}\right)=x_{i, j}$, all the ES turns of $F_{x}, F_{y}^{\mathrm{U}}$ or $F_{y}^{\mathrm{L}}$ that are in $c(F)$ correspond to the NE turns of the corresponding lattice paths $L, L_{1}$ or $L_{2}$. From this, we readily obtain the formula in (i).

(ii) Suppose $i=m$ and $1<j<n-1$. Then for a facet $F=F_{x} F_{y}$ of $\Delta_{0}$ with $\mu\left(F_{x}\right)=x_{m, j}$, the lattice path $L$ corresponding to $F_{x}$ is from $(m, j)$ to $(m, n)$ and evidently this has no NE turns. Consider in $\mathscr{P}((1,2) \rightarrow(i, n),(1,1) \rightarrow(m, j))$ the pair $\left(L_{1}, L_{2}\right)$ corresponding to $\left(F_{y}^{\mathrm{U}}, F_{y}^{\mathrm{L}}\right)$. Suppose the last NE-turn of $L_{1}$ is at $(p, q+1)$. Note that if $q<j$, then we must have $(m, j) \in L_{1}$, which contradicts the fact that $L_{1}, L_{2}$ are nonintersecting. Thus $1 \leq p \leq m-1$ and $j \leq q<n$. Moreover if $q=j$, then by part (iv) of Lemma 11, we see that either $p \leq m-2$ or the NE-turn $(p, q+1)$ is not in $c(F)$. It follows that $L_{1}$ can be replaced by its truncation $\tilde{L}_{1}$, which is a lattice path from $(1,2)$ to $(p, q)$ such that $\tilde{L}_{1}$ and $L_{2}$ are nonintersecting. Moreover the number of NE turns of $\tilde{L}_{1}$ in $c(F)$ are exactly one less than the number of NE turns of $L_{1}$ in $c(F)$, except when $(p, q)=(m-1, j)$ in which case they are the same. Thus by varying $(p, q)$ over an appropriate range, we obtain the formula in (ii).

(iii) If $(i, j)=(m, 1)$ and $F=F_{x} F_{y}$ is a facet of $\Delta_{0}$ with $\mu\left(F_{x}\right)=x_{m, 1}$, then the path $L$ corresponding to $F_{x}$ as well as the path $L_{2}$ corresponding to $F_{y}^{\mathrm{L}}$ have no NE turns. Moreover every NE-turn of the path $L_{1} \in \mathscr{P}((1,2) \rightarrow(m, n))$ corresponding to $F_{y}^{U}$ is necessarily in $c(F)$, thanks to Lemma 11 . Thus, in view of (12), we see that $C_{m, 1}^{s}=\left(\begin{array}{c}n-2 \\ s\end{array}\right)\left(\begin{array}{c}m-1 \\ s\end{array}\right)$. Finally if $(i, j)=(m, n-1)$, then arguing as in (ii) above, we see that for a facet $F=F_{x} F_{y}$ of $\Delta_{0}$ with $\mu\left(F_{x}\right)=x_{m, n-1}$, the lattice path $L$ corresponding to $F_{x}$ has no NE turns and the last NE-turn of the lattice path $L_{1}$ corresponding to $F_{y}^{U}$ must be $(p, n)$ for some $p=1, \ldots, m-1$. Moreover 
by Lemma 11, this turn is counted as a corner (i.e., $x_{p, n} \in c(F)$ ) if and only if $p<m-1$. Thus upon replacing $L_{1}$ by its truncation up to $(p, n-1)$, we obtain the desired formula for $C_{m, n-1}^{s}$ in (iii).

We can already use the results obtained thus far to write down an explicit formula for the Hilbert series of the graded ring $R / \mathscr{I}_{0}$ corresponding to $Z_{0}$. Indeed it suffices to combine Corollary 12, Lemma 13, and Corollary 4 . However the resulting formula is much too complicated and we will instead use results in Section 2 for simplifying various terms in (19) so as to eventually arrive at an elegant formula for (18).

Lemma 14. Let $k$ be a positive integer. Then $C_{m, n-1}^{k}$ is equal to

$$
\sum_{t_{1}+t_{2}=k}\left(\begin{array}{c}
m-2 \\
t_{1}
\end{array}\right)\left(\begin{array}{c}
n-2 \\
t_{1}
\end{array}\right)\left(\begin{array}{c}
m-1 \\
t_{2}
\end{array}\right)\left(\begin{array}{c}
n-2 \\
t_{2}
\end{array}\right)-\left(\begin{array}{c}
m-1 \\
t_{2}+1
\end{array}\right)\left(\begin{array}{c}
n-2 \\
t_{2}
\end{array}\right)\left(\begin{array}{c}
m-2 \\
t_{1}-1
\end{array}\right)\left(\begin{array}{c}
n-2 \\
t_{1}
\end{array}\right) .
$$

Proof. For $s \in \mathbb{Z}$, let $f(s):=\left(\begin{array}{c}m-1 \\ s\end{array}\right)\left(\begin{array}{c}n-2 \\ s\end{array}\right)$ and $g(s):=\left(\begin{array}{c}m-2 \\ s-1\end{array}\right)\left(\begin{array}{c}n-2 \\ s\end{array}\right)$. By Corollary 4,

$$
\begin{aligned}
& \sum_{p=1}^{m-2}\left|\mathscr{P}_{k-1}((1,2) \rightarrow(p, n-1),(1,1) \rightarrow(m, n-1))\right| \\
& \quad=\sum_{p=1}^{m-2} \sum_{s_{1}+s_{2}=k-1}\left(\begin{array}{c}
p-1 \\
s_{1}
\end{array}\right)\left(\begin{array}{c}
n-3 \\
s_{1}
\end{array}\right) f\left(s_{2}\right)-\left(\begin{array}{c}
p \\
s_{2}+1
\end{array}\right)\left(\begin{array}{c}
n-3 \\
s_{2}
\end{array}\right) g\left(s_{1}\right) \\
& \quad=\sum_{s_{1}+s_{2}=k-1}\left(\begin{array}{l}
m-3 \\
\sum^{\prime}=0
\end{array}\left(\begin{array}{c}
p^{\prime} \\
s_{1}
\end{array}\right)\right)\left(\begin{array}{c}
n-3 \\
s_{1}
\end{array}\right) f\left(s_{2}\right)-\left(\sum_{p=1}^{m-2}\left(\begin{array}{c}
p \\
s_{2}+1
\end{array}\right)\right)\left(\begin{array}{c}
n-3 \\
s_{2}
\end{array}\right) g\left(s_{1}\right) \\
& =\sum_{s_{1}+s_{2}=k-1}\left(\begin{array}{c}
m-2 \\
s_{1}+1
\end{array}\right)\left(\begin{array}{c}
n-3 \\
s_{1}
\end{array}\right) f\left(s_{2}\right)-\left(\begin{array}{c}
m-1 \\
s_{2}+2
\end{array}\right)\left(\begin{array}{c}
n-3 \\
s_{2}
\end{array}\right) g\left(s_{1}\right) \\
& \quad=\sum_{t_{1}+t_{2}=k}\left(\begin{array}{c}
m-2 \\
t_{1}
\end{array}\right)\left(\begin{array}{c}
n-3 \\
t_{1}-1
\end{array}\right) f\left(t_{2}\right)-\left(\begin{array}{c}
m-1 \\
t_{2}+1
\end{array}\right)\left(\begin{array}{c}
n-3 \\
t_{2}-1
\end{array}\right) g\left(t_{1}\right),
\end{aligned}
$$

where the penultimate equality follows from Lemma 1 since $\left(\begin{array}{c}0 \\ s_{1}+1\end{array}\right)=0=\left(\begin{array}{c}1 \\ s_{2}+2\end{array}\right)$ for $s_{1}, s_{2} \geq 0$, and also since $\left(\begin{array}{c}n-3 \\ s_{1}\end{array}\right) f\left(s_{2}\right)=0=\left(\begin{array}{c}n-3 \\ s_{2}\end{array}\right) g\left(s_{1}\right)$ if $s_{1}<0$ or $s_{2}<0$, while the last equality follows by altering the summations (twice!) as in (10). On the other hand, by Corollary $4,\left|\mathscr{P}_{k}((1,2) \rightarrow(m-1, n-1),(1,1) \rightarrow(m, n-1))\right|$ is equal to

$$
\sum_{t_{1}+t_{2}=k}\left(\begin{array}{c}
m-2 \\
t_{1}
\end{array}\right)\left(\begin{array}{c}
n-3 \\
t_{1}
\end{array}\right) f\left(t_{2}\right)-\left(\begin{array}{c}
m-1 \\
t_{2}+1
\end{array}\right)\left(\begin{array}{c}
n-3 \\
t_{2}
\end{array}\right) g\left(t_{1}\right) .
$$

Now combining (20) and (21), using (6), and then using part (iii) of Lemma 13, we obtain the desired result. 
Lemma 15. Let $k$ be a positive integer. Then $\sum_{i=1}^{m-1} \sum_{j=1}^{n} C_{i, j}^{k-1}$ is equal to

$$
\sum_{t_{1}+t_{2}=k}\left(\begin{array}{c}
m \\
t_{2}
\end{array}\right)\left(\begin{array}{c}
n \\
t_{1}+1
\end{array}\right)\left(\begin{array}{c}
m-1 \\
t_{1}
\end{array}\right)\left(\begin{array}{c}
n-2 \\
t_{2}-1
\end{array}\right)-\left(\begin{array}{c}
m-1 \\
t_{1}
\end{array}\right)\left(\begin{array}{c}
n \\
t_{2}
\end{array}\right)\left(\begin{array}{c}
m-1 \\
t_{2}-1
\end{array}\right)\left(\begin{array}{c}
n-2 \\
t_{1}
\end{array}\right) .
$$

Proof. Using (12) and part (i) of Lemma 13, we see that $\sum_{i=1}^{m-1} \sum_{j=1}^{n} C_{i, j}^{k-1}$ equals

$$
\sum_{i=1}^{m-1} \sum_{j=1}^{n} \sum_{k_{1}+k_{2}=k-1}\left(\begin{array}{c}
m-i \\
k_{1}
\end{array}\right)\left(\begin{array}{c}
n-j \\
k_{1}
\end{array}\right)\left|\mathscr{P}_{k_{2}}((1,2) \rightarrow(i, n),(1,1) \rightarrow(m, j))\right| .
$$

Applying Corollary 4 and then suitably interchanging summations and noting that the summands below are zero if $k_{1}<0$ or $s_{1}<0$ or $s_{2}<0$, this can be written as

$$
\sum_{\substack{k_{1}+s_{1}+s_{2}=k-1 \\
k_{1}, s_{1}, s_{2} \geq 0}} M_{1} N_{1}\left(\begin{array}{c}
m-1 \\
s_{2}
\end{array}\right)\left(\begin{array}{c}
n-2 \\
s_{1}
\end{array}\right)-M_{2} N_{2}\left(\begin{array}{c}
m-2 \\
s_{1}-1
\end{array}\right)\left(\begin{array}{c}
n-2 \\
s_{2}
\end{array}\right),
$$

where, for any given $k_{1}, s_{1}, s_{2} \geq 0$, we have temporarily put

$$
\begin{aligned}
M_{1}=\sum_{i=1}^{m-1}\left(\begin{array}{c}
m-i \\
k_{1}
\end{array}\right)\left(\begin{array}{c}
i-1 \\
s_{1}
\end{array}\right), & N_{1}=\sum_{j=1}^{n}\left(\begin{array}{c}
n-j \\
k_{1}
\end{array}\right)\left(\begin{array}{c}
j-1 \\
s_{2}
\end{array}\right)=\left(\begin{array}{c}
n \\
k_{1}+s_{2}+1
\end{array}\right), \\
M_{2}=\sum_{i=1}^{m-1}\left(\begin{array}{c}
m-i \\
k_{1}
\end{array}\right)\left(\begin{array}{c}
i \\
s_{2}+1
\end{array}\right), & N_{2}=\sum_{j=1}^{n}\left(\begin{array}{c}
n-j \\
k_{1}
\end{array}\right)\left(\begin{array}{c}
j-1 \\
s_{1}
\end{array}\right)=\left(\begin{array}{c}
n \\
k_{1}+s_{1}+1
\end{array}\right),
\end{aligned}
$$

and where the simplified expressions for $N_{1}, N_{2}$ follow by rewriting each of the summands in $N_{1}$ and $N_{2}$ using (5), invoking (4) (noting that $k_{1}, s_{1}, s_{2} \geq 0$ ), and then applying (9) for suitable values of $s, t, \alpha$ and $\beta$. A similar simplification is possible in $M_{1}$ and $M_{2}$ if we add and subtract the term corresponding to $i=m$, and in view of (4), this is only necessary if $k_{1}=0$. Thus

$$
M_{1}=\left(\begin{array}{c}
m \\
k_{1}+s_{1}+1
\end{array}\right)-\delta_{0, k_{1}}\left(\begin{array}{c}
m-1 \\
s_{1}
\end{array}\right) \quad \text { and } \quad M_{2}=\left(\begin{array}{c}
m+1 \\
k_{1}+s_{2}+2
\end{array}\right)-\delta_{0, k_{1}}\left(\begin{array}{c}
m \\
s_{2}+1
\end{array}\right)
$$

where $\delta$ is the Kronecker delta. Substituting the simplified values of $M_{1}, N_{1}, M_{2}, N_{2}$ in (22), and letting

$$
A\left(s_{1}, s_{2}\right):=\left(\begin{array}{c}
m-1 \\
s_{2}
\end{array}\right)\left(\begin{array}{c}
n-2 \\
s_{1}
\end{array}\right), \quad B\left(s_{1}, s_{2}\right):=\left(\begin{array}{c}
m-2 \\
s_{1}-1
\end{array}\right)\left(\begin{array}{c}
n-2 \\
s_{2}
\end{array}\right)
$$

for $s_{1}, s_{2} \in \mathbb{Z}$, we see that (22) is of the form $E_{3}+S_{3}$, where

$$
E_{3}=\sum_{\substack{k_{1}+s_{1}+s_{2}=k-1 \\
k_{1}, s_{1}, s_{2} \geq 0}}\left(\begin{array}{c}
m \\
k-s_{2}
\end{array}\right)\left(\begin{array}{c}
n \\
k-s_{1}
\end{array}\right) A\left(s_{1}, s_{2}\right)-\left(\begin{array}{c}
m+1 \\
k-s_{1}+1
\end{array}\right)\left(\begin{array}{c}
n \\
k-s_{2}
\end{array}\right) B\left(s_{1}, s_{2}\right),
$$

and $S_{3}$ is the part where the Kronecker delta is nonzero: 


$$
S_{3}=\sum_{s_{1}+s_{2}=k-1}\left(\begin{array}{c}
m \\
s_{2}+1
\end{array}\right)\left(\begin{array}{c}
n \\
s_{1}+1
\end{array}\right) B\left(s_{1}, s_{2}\right)-\left(\begin{array}{c}
m-1 \\
s_{1}
\end{array}\right)\left(\begin{array}{c}
n \\
s_{2}+1
\end{array}\right) A\left(s_{1}, s_{2}\right) .
$$

Altering the summation as in (10), we see that $S_{3}$ can be written as

$$
\sum_{t_{1}+t_{2}=k}\left(\begin{array}{c}
m \\
t_{2}
\end{array}\right)\left(\begin{array}{c}
n \\
t_{1}+1
\end{array}\right)\left(\begin{array}{c}
m-2 \\
t_{1}-1
\end{array}\right)\left(\begin{array}{c}
n-2 \\
t_{2}-1
\end{array}\right)-\left(\begin{array}{c}
m-1 \\
t_{1}
\end{array}\right)\left(\begin{array}{c}
n \\
t_{2}
\end{array}\right)\left(\begin{array}{c}
m-1 \\
t_{2}-1
\end{array}\right)\left(\begin{array}{c}
n-2 \\
t_{1}
\end{array}\right) .
$$

On the other hand, in view of (4) and (11), we can write

$$
E_{3}=\sum_{\ell=0}^{k-1} \sum_{s_{1}+s_{2}=\ell}\left(\begin{array}{c}
m \\
k-s_{1}
\end{array}\right)\left(\begin{array}{c}
n \\
k-s_{2}
\end{array}\right) A\left(s_{2}, s_{1}\right)-\left(\begin{array}{c}
m+1 \\
k-s_{1}+1
\end{array}\right)\left(\begin{array}{c}
n \\
k-s_{2}
\end{array}\right) B\left(s_{1}, s_{2}\right) .
$$

By (6), we have

$$
\left(\begin{array}{c}
m+1 \\
k-s_{1}+1
\end{array}\right)=\left(\begin{array}{c}
m \\
k-s_{1}
\end{array}\right)+\left(\begin{array}{c}
m \\
k-\left(s_{1}-1\right)
\end{array}\right) .
$$

Using this to split the second summand in $E_{3}$ into two parts and combining one of the parts with the first summand in $E_{3}$ and then applying (6) once again, we see that

$$
E_{3}=\sum_{\ell=0}^{k-1} \sum_{s_{1}+s_{2}=\ell} f\left(s_{1}, s_{2}\right)-f\left(s_{1}-1, s_{2}\right)
$$

where

$$
f\left(s_{1}, s_{2}\right):=\left(\begin{array}{c}
m \\
k-s_{1}
\end{array}\right)\left(\begin{array}{c}
n \\
k-s_{2}
\end{array}\right)\left(\begin{array}{c}
m-2 \\
s_{1}
\end{array}\right)\left(\begin{array}{c}
n-2 \\
s_{2}
\end{array}\right)
$$

for $s_{1}, s_{2} \in \mathbb{Z}$. Now in view of (10), we find that $E_{3}$ is given by the telescoping sum

$$
E_{3}=\sum_{\ell=0}^{k-1} F_{\ell}-F_{\ell-1}, \quad \text { where } F_{\ell}:=\sum_{s_{1}+s_{2}=\ell} f\left(s_{1}, s_{2}\right) \text { for } \ell \in \mathbb{Z} .
$$

From the definition of $f$, we see that $F_{-1}=0$, and thus $E_{3}=F_{k-1}$, that is,

$$
E_{3}=\sum_{s_{1}+s_{2}=k-1}\left(\begin{array}{c}
m \\
k-s_{1}
\end{array}\right)\left(\begin{array}{c}
n \\
k-s_{2}
\end{array}\right)\left(\begin{array}{c}
m-2 \\
s_{1}
\end{array}\right)\left(\begin{array}{c}
n-2 \\
s_{2}
\end{array}\right) .
$$

Now we can replace $k-s_{1}, k-s_{2}$ by $s_{2}+1, s_{1}+1$, respectively, in the above summand, and then alter the summation using (10) to obtain

$$
E_{3}=\sum_{t_{1}+t_{2}=k}\left(\begin{array}{c}
m \\
t_{2}
\end{array}\right)\left(\begin{array}{c}
n \\
t_{1}+1
\end{array}\right)\left(\begin{array}{c}
m-2 \\
t_{1}
\end{array}\right)\left(\begin{array}{c}
n-2 \\
t_{2}-1
\end{array}\right) .
$$

Finally, by adding (24) and (23) termwise and using (6), we obtain the desired formula for $E_{3}+S_{3}$, i.e., for $\sum_{i=1}^{m-1} \sum_{j=1}^{n} C_{i, j}^{k-1}$. 
Lemma 16. Let $k$ be a positive integer. Then $\sum_{j=1}^{n-2} C_{m, j}^{k-1}$ is equal to

$$
\sum_{t_{1}+t_{2}=k}\left(\begin{array}{c}
m-1 \\
t_{1}
\end{array}\right)\left(\begin{array}{c}
n-2 \\
t_{1}
\end{array}\right)\left(\begin{array}{c}
m-1 \\
t_{2}-1
\end{array}\right)\left(\begin{array}{c}
n-2 \\
t_{2}
\end{array}\right)-\left(\begin{array}{c}
m \\
t_{2}+1
\end{array}\right)\left(\begin{array}{c}
n-2 \\
t_{2}
\end{array}\right)\left(\begin{array}{c}
m-2 \\
t_{1}-2
\end{array}\right)\left(\begin{array}{c}
n-2 \\
t_{1}
\end{array}\right)
$$

Proof. The desired result is easily verified when $n \leq 3$ and so we assume that $n>3$. For $j, s \in \mathbb{Z}$, let

$$
f_{j}(s):=\left(\begin{array}{c}
m-1 \\
s
\end{array}\right)\left(\begin{array}{c}
j-1 \\
s
\end{array}\right), \quad g_{j}(s):=\left(\begin{array}{c}
m-2 \\
s-1
\end{array}\right)\left(\begin{array}{c}
j-1 \\
s
\end{array}\right) .
$$

In view of parts (iii) and (ii) of Lemma 13 together with (4) and Corollary 4, we see that

$$
C_{m, 1}^{k-1}=\left(\begin{array}{c}
n-2 \\
k-1
\end{array}\right)\left(\begin{array}{c}
m-1 \\
k-1
\end{array}\right) \quad \text { and } \quad \sum_{j=2}^{n-2} C_{m, j}^{k-1}=S_{4}+S_{5}+S_{6}
$$

where

$$
\begin{aligned}
& S_{4}=\sum_{j=2}^{n-2} \sum_{p=1}^{m-1} \sum_{\substack{q=j+1 \\
s_{1}+s_{2}=k-2 \\
s_{1}, s_{2} \geq 0}}^{n-1}\left(\begin{array}{c}
p-1 \\
s_{1}
\end{array}\right)\left(\begin{array}{c}
q-2 \\
s_{1}
\end{array}\right) f_{j}\left(s_{2}\right)-\left(\begin{array}{c}
p \\
s_{2}+1
\end{array}\right)\left(\begin{array}{c}
q-2 \\
s_{2}
\end{array}\right) g_{j}\left(s_{1}\right), \\
& S_{5}=\sum_{j=2}^{n-2} \sum_{p=1}^{m-2} \sum_{\substack{s_{1}+s_{2}=k-2 \\
s_{1}, s_{2} \geq 0}}\left(\begin{array}{c}
p-1 \\
s_{1}
\end{array}\right)\left(\begin{array}{c}
j-2 \\
s_{1}
\end{array}\right) f_{j}\left(s_{2}\right)-\left(\begin{array}{c}
p \\
s_{2}+1
\end{array}\right)\left(\begin{array}{c}
j-2 \\
s_{2}
\end{array}\right) g_{j}\left(s_{1}\right), \\
& S_{6}=\sum_{j=2}^{n-2} \sum_{s_{1}+s_{2}=k-1}\left(\begin{array}{c}
m-2 \\
s_{1}
\end{array}\right)\left(\begin{array}{c}
j-2 \\
s_{1}
\end{array}\right) f_{j}\left(s_{2}\right)-\left(\begin{array}{c}
m-1 \\
s_{2}+1
\end{array}\right)\left(\begin{array}{c}
j-2 \\
s_{2}
\end{array}\right) g_{j}\left(s_{1}\right) .
\end{aligned}
$$

Interchanging $s_{1}$ and $s_{2}$ in the second summand for $S_{6}$ as in (11), we can write

$$
S_{6}=\sum_{s_{1}+s_{2}=k-1} \lambda\left(s_{1}, s_{2}\right)\left(\left(\begin{array}{c}
m-2 \\
s_{1}
\end{array}\right)\left(\begin{array}{c}
m-1 \\
s_{2}
\end{array}\right)-\left(\begin{array}{c}
m-1 \\
s_{1}+1
\end{array}\right)\left(\begin{array}{c}
m-2 \\
s_{2}-1
\end{array}\right)\right),
$$

where, for $s_{1}, s_{2} \in \mathbb{Z}$, we let

$$
\lambda\left(s_{1}, s_{2}\right):=\sum_{j=2}^{n-2}\left(\begin{array}{c}
j-2 \\
s_{1}
\end{array}\right)\left(\begin{array}{c}
j-1 \\
s_{2}
\end{array}\right) .
$$

Next, by Lemma 1,

$$
\sum_{p=1}^{m-2}\left(\begin{array}{c}
p-1 \\
s_{1}
\end{array}\right)=\left(\begin{array}{c}
m-2 \\
s_{1}+1
\end{array}\right) \quad \text { and } \quad \sum_{p=1}^{m-2}\left(\begin{array}{c}
p \\
s_{2}+1
\end{array}\right)=\left(\begin{array}{c}
m-1 \\
s_{2}+2
\end{array}\right) \quad \text { for } s_{1}, s_{2} \geq 0 .
$$

Consequently, by interchanging summations and rearranging terms, we find 


$$
\begin{aligned}
S_{5} & =\sum_{j=2}^{n-2} \sum_{\substack{s_{1}+s_{2}=k-2 \\
s_{1}, s_{2} \geq 0}}\left(\begin{array}{c}
m-2 \\
s_{1}+1
\end{array}\right)\left(\begin{array}{c}
j-2 \\
s_{1}
\end{array}\right) f_{j}\left(s_{2}\right)-\left(\begin{array}{c}
m-1 \\
s_{2}+2
\end{array}\right)\left(\begin{array}{c}
j-2 \\
s_{2}
\end{array}\right) g_{j}\left(s_{1}\right) \\
& =\sum_{s_{1}+s_{2}=k-2} \lambda\left(s_{1}, s_{2}\right)\left(\left(\begin{array}{c}
m-2 \\
s_{1}+1
\end{array}\right)\left(\begin{array}{c}
m-1 \\
s_{2}
\end{array}\right)-\left(\begin{array}{c}
m-1 \\
s_{1}+2
\end{array}\right)\left(\begin{array}{c}
m-2 \\
s_{2}-1
\end{array}\right)\right) \\
& =\sum_{s_{1}+s_{2}=k-1} \lambda\left(s_{1}-1, s_{2}\right)\left(\left(\begin{array}{c}
m-2 \\
s_{1}
\end{array}\right)\left(\begin{array}{c}
m-1 \\
s_{2}
\end{array}\right)-\left(\begin{array}{c}
m-1 \\
s_{1}+1
\end{array}\right)\left(\begin{array}{c}
m-2 \\
s_{2}-1
\end{array}\right)\right),
\end{aligned}
$$

where the penultimate equality follows from (4) and (11) by interchanging $s_{1}$ and $s_{2}$ in the second summand of the preceding formula, while the last equality follows from (10). Now, using (6), we easily see that

$$
\lambda\left(s_{1}-1, s_{2}\right)+\lambda\left(s_{1}, s_{2}\right)=v\left(s_{1}, s_{2}\right) \quad \text { for any } s_{1}, s_{2} \in \mathbb{Z},
$$

where

$$
v\left(s_{1}, s_{2}\right):=\sum_{j=2}^{n-2}\left(\begin{array}{c}
j-1 \\
s_{1}
\end{array}\right)\left(\begin{array}{c}
j-1 \\
s_{2}
\end{array}\right) .
$$

Hence we can combine (27) and (26) to obtain

$$
S_{5}+S_{6}=\sum_{s_{1}+s_{2}=k-1} v\left(s_{1}, s_{2}\right)\left(\left(\begin{array}{c}
m-2 \\
s_{1}
\end{array}\right)\left(\begin{array}{c}
m-1 \\
s_{2}
\end{array}\right)-\left(\begin{array}{c}
m-1 \\
s_{1}+1
\end{array}\right)\left(\begin{array}{c}
m-2 \\
s_{2}-1
\end{array}\right)\right) .
$$

It remains to consider $S_{4}$ or rather $C_{m, 1}^{k-1}+S_{4}$. This is a little more complicated, but it can be handled using arguments similar to those in the proof of Lemma 15 as follows. First, by interchanging summations and using Lemma 1, we find

$$
S_{4}=\sum_{j=2}^{n-2} \sum_{\substack{s_{1}+s_{2}=k-2 \\
s_{1}, s_{2} \geq 0}}\left(\begin{array}{c}
m-1 \\
s_{1}+1
\end{array}\right) \theta\left(s_{1}\right) f_{j}\left(s_{2}\right)-\left(\begin{array}{c}
m \\
s_{2}+2
\end{array}\right) \theta\left(s_{2}\right) g_{j}\left(s_{1}\right),
$$

where, for $s \in \mathbb{Z}$, we have let

$$
\theta(s):=\left(\begin{array}{c}
n-2 \\
s+1
\end{array}\right)-\left(\begin{array}{c}
j-1 \\
s+1
\end{array}\right) .
$$

Now observe that if $s_{1}<0$ or $s_{2}<0$, then $\theta\left(s_{1}\right) f_{j}\left(s_{2}\right)=0=\theta\left(s_{2}\right) g_{j}\left(s_{1}\right)$. Thus we may drop the condition $s_{1}, s_{2} \geq 0$ in the above expression for $S_{4}$, and then alter each of the two summations over $\left(s_{1}, s_{2}\right)$ using (10) to write

$$
S_{4}=\sum_{j=2}^{n-2} \sum_{s_{1}+s_{2}=k-1}\left(\begin{array}{c}
m-1 \\
s_{1}
\end{array}\right) \theta\left(s_{1}-1\right) f_{j}\left(s_{2}\right)-\left(\begin{array}{c}
m \\
s_{2}+1
\end{array}\right) \theta\left(s_{2}-1\right) g_{j}\left(s_{1}\right) .
$$

Next we collate the terms involving $j$ and bring the summation over $j$ inside, and 
note that, by Lemma $1, \sum_{j=2}^{n-2}\left(\begin{array}{c}j-1 \\ s\end{array}\right)=\left(\begin{array}{c}n-2 \\ s+1\end{array}\right)-\delta_{0, s}$ for any $s \geq 0$. This yields

$$
\begin{aligned}
S_{4}=\sum_{s_{1}+s_{2}=k-1} & \left(\begin{array}{c}
m-1 \\
s_{1}
\end{array}\right)\left(\begin{array}{c}
n-2 \\
s_{1}
\end{array}\right)\left(\begin{array}{c}
m-1 \\
s_{2}
\end{array}\right)\left(\left(\begin{array}{c}
n-2 \\
s_{2}+1
\end{array}\right)-\delta_{0, s_{2}}\right) \\
& -\left(\begin{array}{c}
m \\
s_{2}+1
\end{array}\right)\left(\begin{array}{c}
n-2 \\
s_{2}
\end{array}\right)\left(\begin{array}{c}
m-2 \\
s_{1}-1
\end{array}\right)\left(\left(\begin{array}{c}
n-2 \\
s_{1}+1
\end{array}\right)-\delta_{0, s_{1}}\right) \\
& -\left(\begin{array}{c}
m-1 \\
s_{1}
\end{array}\right)\left(\begin{array}{c}
m-1 \\
s_{2}
\end{array}\right) v\left(s_{1}, s_{2}\right)+\left(\begin{array}{c}
m \\
s_{2}+1
\end{array}\right)\left(\begin{array}{c}
m-2 \\
s_{1}-1
\end{array}\right) v\left(s_{1}, s_{2}\right) .
\end{aligned}
$$

Since $\left(\begin{array}{c}m-2 \\ s_{1}-1\end{array}\right)=0$ when $s_{1}=0$, the only contribution of the terms involving Kronecker delta is when $s_{2}=0$, and it is $-\left(\begin{array}{c}m-1 \\ k-1\end{array}\right)\left(\begin{array}{c}n-2 \\ k-1\end{array}\right)$, that is, precisely $-C_{m, 1}^{k-1}$. It follows that $C_{m, 1}^{k-1}+S_{4}=S_{4}^{*}+E_{4}$, where

$$
S_{4}^{*}=\sum_{s_{1}+s_{2}=k-1}\left(\begin{array}{c}
m-1 \\
s_{1}
\end{array}\right)\left(\begin{array}{c}
n-2 \\
s_{1}
\end{array}\right)\left(\begin{array}{c}
m-1 \\
s_{2}
\end{array}\right)\left(\begin{array}{c}
n-2 \\
s_{2}+1
\end{array}\right)-\left(\begin{array}{c}
m \\
s_{2}+1
\end{array}\right)\left(\begin{array}{c}
n-2 \\
s_{2}
\end{array}\right)\left(\begin{array}{c}
m-2 \\
s_{1}-1
\end{array}\right)\left(\begin{array}{c}
n-2 \\
s_{1}+1
\end{array}\right)
$$

and

$$
\begin{aligned}
E_{4} & =\sum_{s_{1}+s_{2}=k-1} v\left(s_{1}, s_{2}\right)\left(\left(\begin{array}{c}
m \\
s_{2}+1
\end{array}\right)\left(\begin{array}{c}
m-2 \\
s_{1}-1
\end{array}\right)-\left(\begin{array}{c}
m-1 \\
s_{1}
\end{array}\right)\left(\begin{array}{c}
m-1 \\
s_{2}
\end{array}\right)\right) \\
& =\sum_{s_{1}+s_{2}=k-1} v\left(s_{1}, s_{2}\right)\left(\left(\begin{array}{c}
m \\
s_{1}+1
\end{array}\right)\left(\begin{array}{c}
m-2 \\
s_{2}-1
\end{array}\right)-\left(\begin{array}{c}
m-1 \\
s_{1}
\end{array}\right)\left(\begin{array}{c}
m-1 \\
s_{2}
\end{array}\right)\right),
\end{aligned}
$$

where the last equality follows by interchanging $s_{1}$ and $s_{2}$, while noting that $v$ is symmetric in $s_{1}, s_{2}$.

Now combining (28) and (29), and then, making an easy calculation using (6), we see that

$$
E_{4}+S_{5}+S_{6}=\sum_{s_{1}+s_{2}=k-1} v\left(s_{1}, s_{2}\right)\left(\left(\begin{array}{c}
m-1 \\
s_{1}
\end{array}\right)\left(\begin{array}{c}
m-2 \\
s_{2}-1
\end{array}\right)-\left(\begin{array}{c}
m-2 \\
s_{1}-1
\end{array}\right)\left(\begin{array}{c}
m-1 \\
s_{2}
\end{array}\right)\right)=0
$$

where the last equality follows by interchanging $s_{1}$ and $s_{2}$ in one of the summations above. Thus $\sum_{j=1}^{n-2} C_{m, j}^{k-1}=S_{4}^{*}$. Finally, using (10), we readily see that $S_{4}^{*}$ is precisely the desired formula in the statement of the lemma.

Corollary 17. Let $k$ be a positive integer. Then $C_{m, n-1}^{k}+\sum_{j=1}^{n-2} C_{m, j}^{k-1}$ is equal to

$$
\sum_{t_{1}+t_{2}=k}\left(\begin{array}{c}
m-1 \\
t_{1}
\end{array}\right)\left(\begin{array}{c}
n-2 \\
t_{1}
\end{array}\right)\left(\begin{array}{c}
m-1 \\
t_{2}
\end{array}\right)\left(\begin{array}{c}
n-2 \\
t_{2}
\end{array}\right)-\left(\begin{array}{c}
m-1 \\
t_{2}+1
\end{array}\right)\left(\begin{array}{c}
n-2 \\
t_{2}
\end{array}\right)\left(\begin{array}{c}
m-1 \\
t_{1}-1
\end{array}\right)\left(\begin{array}{c}
n-2 \\
t_{1}
\end{array}\right) .
$$

Proof. Consider the formula for $\sum_{j=1}^{n-2} C_{m, j}^{k-1}$ given by Lemma 16. This is a difference 
of two summations over $\left(t_{1}, t_{2}\right) \in \mathbb{Z}^{2}$ with $t_{1}+t_{2}=k$. Alter the first of these summations by interchanging $t_{1}$ and $t_{2}$, while putting $\left(\begin{array}{c}m \\ t_{2}+1\end{array}\right)=\left(\begin{array}{c}m-1 \\ t_{2}\end{array}\right)+\left(\begin{array}{c}m-1 \\ t_{2}+1\end{array}\right)$ in the second summation to split it into two summations. Then, using (6), we readily see that the formula for $\sum_{j=1}^{n-2} C_{m, j}^{k-1}$ becomes

$$
\sum_{t_{1}+t_{2}=k}\left(\begin{array}{c}
m-2 \\
t_{1}-1
\end{array}\right)\left(\begin{array}{c}
n-2 \\
t_{1}
\end{array}\right)\left(\begin{array}{c}
m-1 \\
t_{2}
\end{array}\right)\left(\begin{array}{c}
n-2 \\
t_{2}
\end{array}\right)-\left(\begin{array}{c}
m-1 \\
t_{2}+1
\end{array}\right)\left(\begin{array}{c}
n-2 \\
t_{2}
\end{array}\right)\left(\begin{array}{c}
m-2 \\
t_{1}-2
\end{array}\right)\left(\begin{array}{c}
n-2 \\
t_{1}
\end{array}\right) .
$$

This can be added termwise, using (6) once again, with the formula for $C_{m, n-1}^{k}$ given by Lemma 14, to obtain the desired result.

We are now ready for our main theorem.

Theorem 18. The Hilbert series of $R / \Phi_{0}$ is given by

$$
\left(\frac{\sum_{e=0}^{m-1}\left(\begin{array}{c}
m-1 \\
e
\end{array}\right)\left(\begin{array}{c}
n-1 \\
e
\end{array}\right) z^{e}}{(1-z)^{m+n-1}}\right)^{2}
$$

Proof. First note that (30) is of the form $(1-z)^{-2(m+n-1)} \sum_{k=0}^{2 m-2} h_{k}^{*} z^{k}$, where

$$
h_{k}^{*}=\sum_{t_{1}+t_{2}=k}\left(\begin{array}{c}
m-1 \\
t_{1}
\end{array}\right)\left(\begin{array}{c}
n-1 \\
t_{1}
\end{array}\right)\left(\begin{array}{c}
m-1 \\
t_{2}
\end{array}\right)\left(\begin{array}{c}
n-1 \\
t_{2}
\end{array}\right) \quad \text { for } k \in \mathbb{Z} .
$$

On the other hand, by Corollary 12, we see that the Hilbert series of $R / \Phi_{0}$ is given by $(1-z)^{-2(m+n-1)} \sum_{k \geq 0} h_{k} z^{k}$, where $h_{0}=1$, and

$$
h_{k}=\left(C_{m, n-1}^{k}+\sum_{j=1}^{n-2} C_{m, j}^{k-1}\right)+\sum_{i=1}^{m-1} \sum_{j=1}^{n} C_{i, j}^{k-1} \quad \text { for } k \geq 1 .
$$

It is clear that $h_{0}^{*}=1=h_{0}$ and so it suffices to show that $h_{k}^{*}=h_{k}$ for all $k \geq 1$. In view of Corollary 17 and Lemma 15, this is equivalent to showing that

$$
\sum_{t_{1}+t_{2}=k} P_{1}\left(t_{1}, t_{2}\right)-P_{2}\left(t_{1}, t_{2}\right)+P_{3}\left(t_{1}, t_{2}\right)-P_{4}\left(t_{1}, t_{2}\right)-P\left(t_{1}, t_{2}\right)=0 \quad \text { for } k \geq 1 \text {, }
$$

where $P_{i}\left(t_{1}, t_{2}\right)$ for $i=1, \ldots, 4$, and $P\left(t_{1}, t_{2}\right)$ are the relevant summands, namely,

$$
\begin{aligned}
& P_{1}\left(t_{1}, t_{2}\right):=\left(\begin{array}{c}
m-1 \\
t_{1}
\end{array}\right)\left(\begin{array}{c}
n-2 \\
t_{1}
\end{array}\right)\left(\begin{array}{c}
m-1 \\
t_{2}
\end{array}\right)\left(\begin{array}{c}
n-2 \\
t_{2}
\end{array}\right), \\
& P_{2}\left(t_{1}, t_{2}\right):=\left(\begin{array}{c}
m-1 \\
t_{2}+1
\end{array}\right)\left(\begin{array}{c}
n-2 \\
t_{2}
\end{array}\right)\left(\begin{array}{c}
m-1 \\
t_{1}-1
\end{array}\right)\left(\begin{array}{c}
n-2 \\
t_{1}
\end{array}\right), \\
& P_{3}\left(t_{1}, t_{2}\right):=\left(\begin{array}{c}
m \\
t_{2}
\end{array}\right)\left(\begin{array}{c}
n \\
t_{1}+1
\end{array}\right)\left(\begin{array}{c}
m-1 \\
t_{1}
\end{array}\right)\left(\begin{array}{c}
n-2 \\
t_{2}-1
\end{array}\right), \\
& P_{4}\left(t_{1}, t_{2}\right):=\left(\begin{array}{c}
m-1 \\
t_{1}
\end{array}\right)\left(\begin{array}{c}
n \\
t_{2}
\end{array}\right)\left(\begin{array}{c}
m-1 \\
t_{2}-1
\end{array}\right)\left(\begin{array}{c}
n-2 \\
t_{1}
\end{array}\right),
\end{aligned}
$$


and

$$
P\left(t_{1}, t_{2}\right):=\left(\begin{array}{c}
m-1 \\
t_{1}
\end{array}\right)\left(\begin{array}{c}
n-1 \\
t_{1}
\end{array}\right)\left(\begin{array}{c}
m-1 \\
t_{2}
\end{array}\right)\left(\begin{array}{c}
n-1 \\
t_{2}
\end{array}\right)
$$

for $t_{1}, t_{2} \in \mathbb{Z}$. To this end, we will make an extensive use of alterations as in (10) and (11); more specifically, the fact that

$$
\sum_{t_{1}+t_{2}=k} f\left(t_{1}, t_{2}\right)=\sum_{t_{1}+t_{2}=k} f\left(t_{2}, t_{1}\right)=\sum_{t_{1}+t_{2}=k} f\left(t_{1}+1, t_{2}-1\right)=\sum_{t_{1}+t_{2}=k} f\left(t_{2}+1, t_{1}-1\right)
$$

for any $f: \mathbb{Z}^{2} \rightarrow \mathbb{Q}$ with finite support and any $k \in \mathbb{Z}$. Now fix any positive integer $k$ and any $\left(t_{1}, t_{2}\right) \in \mathbb{Z}^{2}$ with $t_{1}+t_{2}=k$. Observe that

$$
P_{3}\left(t_{1}-1, t_{2}+1\right)-P_{4}\left(t_{2}, t_{1}\right)=\left(\begin{array}{c}
m-1 \\
t_{2}+1
\end{array}\right)\left(\begin{array}{c}
n \\
t_{1}
\end{array}\right)\left(\begin{array}{c}
m-1 \\
t_{1}-1
\end{array}\right)\left(\begin{array}{c}
n-2 \\
t_{2}
\end{array}\right) .
$$

Using (6) twice, we may substitute $\left(\begin{array}{c}n-2 \\ t_{1}\end{array}\right)+\left(\begin{array}{c}n-2 \\ t_{1}-1\end{array}\right)+\left(\begin{array}{c}n-1 \\ t_{1}-1\end{array}\right)$ for $\left(\begin{array}{c}n \\ t_{1}\end{array}\right)$ in the right-hand side of the above identity to obtain

$$
-P_{2}\left(t_{1}, t_{2}\right)+P_{3}\left(t_{1}-1, t_{2}+1\right)-P_{4}\left(t_{2}, t_{1}\right)=Q_{1}\left(t_{1}, t_{2}\right)+Q_{2}\left(t_{1}, t_{2}\right),
$$

where

$$
\begin{aligned}
Q_{1}\left(t_{1}, t_{2}\right) & :=\left(\begin{array}{l}
m-1 \\
t_{2}+1
\end{array}\right)\left(\begin{array}{c}
n-2 \\
t_{1}-1
\end{array}\right)\left(\begin{array}{c}
m-1 \\
t_{1}-1
\end{array}\right)\left(\begin{array}{c}
n-2 \\
t_{2}
\end{array}\right), \\
Q_{2}\left(t_{1}, t_{2}\right) & :=\left(\begin{array}{c}
m-1 \\
t_{2}+1
\end{array}\right)\left(\begin{array}{c}
n-1 \\
t_{1}-1
\end{array}\right)\left(\begin{array}{c}
m-1 \\
t_{1}-1
\end{array}\right)\left(\begin{array}{c}
n-2 \\
t_{2}
\end{array}\right) .
\end{aligned}
$$

Finally observe that $P_{1}\left(t_{1}, t_{2}\right)+Q_{1}\left(t_{1}+1, t_{2}-1\right)+Q_{2}\left(t_{2}+1, t_{1}-1\right)=P\left(t_{1}, t_{2}\right)$. This yields the desired result.

It may be noted that in view of (2) and (30), the Hilbert series of the principal component $Z_{0}$ is precisely the square of the Hilbert series of the base variety $\mathscr{E}_{2}^{m, n}$, and, as such, Theorem 7 could be deduced as a consequence of Theorem 18 .

As an application of Theorem 18, we will now compute the $a$-invariant of the coordinate ring $R / \mathscr{I}_{0}$ of the principal component $Z_{0}$ of $\mathscr{E}_{2,2}^{m, n}$ and determine when $Z_{0}$ is Gorenstein. Recall that if $A$ is a finitely generated, positively graded CohenMacaulay algebra over a field, then $A$ admits a graded canonical module $\omega_{A}$ and the $a$-invariant of $A$ is defined as the negative of the least degree of a generator of $\omega_{A}$. If the Hilbert series of $A$ is given by $H_{A}(z)=h(z) /(1-z)^{d}$, where $d=\operatorname{dim} A$ and $h(z) \in \mathbb{Q}[z]$ with $h(1) \neq 0$, then the $a$-invariant of $A$ is the order of the pole of $H_{A}(z)$ at infinity, which is $-(d-\operatorname{deg} h(z))$. Moreover the Hilbert series of $\omega_{A}$ is given by $H_{\omega_{A}}(z)=(-1)^{d} H_{A}\left(z^{-1}\right)$. As a general reference for these notions and results, one may consult [Bruns and Herzog 1993], especially Sections 3.6 and 4.4. The following result is an analogue of a theorem of Gräbe [1988] (see also 
[Ghorpade 1996, Theorem 4]) for classical determinantal varieties which says that if $1 \leq r \leq m \leq n$, then the $a$-invariant of (the coordinate ring of) $\mathscr{E}_{r}^{m, n}$ is $-(r-1) n$.

Corollary 19. The a-invariant of $R / \Phi_{0}$ is equal to $-2 n$ and the Hilbert series of the graded canonical module of $R / \mathscr{I}_{0}$ is given by

$$
\left(\frac{\sum_{e=0}^{m-1}\left(\begin{array}{c}
m-1 \\
e
\end{array}\right)\left(\begin{array}{c}
n-1 \\
e
\end{array}\right) z^{m+n-e-1}}{(1-z)^{m+n-1}}\right)^{2} .
$$

Proof. We know from [Jonov 2011, Theorem 1.2] that $A=R / I_{0}$ is Cohen-Macaulay and it is obviously a finitely generated, positively graded $\mathbb{F}$-algebra. Moreover, by Theorem 18, the Hilbert series of $A$ is given by $h_{0}(z) /(1-z)^{2(m+n-1)}$, where

$$
h_{0}(z)=\left(\sum_{e=0}^{m-1}\left(\begin{array}{c}
m-1 \\
e
\end{array}\right)\left(\begin{array}{c}
n-1 \\
e
\end{array}\right) z^{e}\right)^{2} .
$$

Since $2 \leq m \leq n$, we see that $h_{0}(z)$ is a polynomial in $z$ of degree $2(m-1)$, with leading coefficient $\left(\begin{array}{c}n-1 \\ m-1\end{array}\right)^{2}$, and all other coefficients nonnegative integers; in particular, $h_{0}(1) \neq 0$. Hence the $a$-invariant of $A=R / \mathscr{I}_{0}$ is

$$
2(m-1)-2(m+n-1)=-2 n,
$$

and also that the Hilbert series of $\omega_{A}$ is given by (33).

The following result is an analogue of a theorem of Svanes [1974] (see also [Conca and Herzog 1994]) for classical determinantal varieties which says that for any $r \geq 1$, (the coordinate ring of) $\mathscr{E}_{r}^{m, n}$ is Gorenstein if and only if $m=n$.

Corollary 20. The coordinate ring $R / \mathscr{I}_{0}$ of $Z_{0}$ is Gorenstein if and only if $m=n$.

Proof. By [Jonov 2011, Theorem 1.2] and [Košir and Sethuraman 2005b, Proposition 3.3], $A=R / \mathscr{I}_{0}$ is a Cohen-Macaulay domain. Hence from a well-known result of Stanley [1978, Theorem 4.4] (see also [Bruns and Herzog 1993, Corollary 4.4.6]), we see that $A$ is Gorenstein if and only if $H_{A}(z)=(-1)^{d} z^{a} H_{A}\left(z^{-1}\right)$ for some $a \in \mathbb{Z}$. Moreover, in this case, the integer $a$ is necessarily the $a$-invariant of $A$. Thus, from Corollary 19 , we see that $R / \mathscr{I}_{0}$ is Gorenstein if and only if

$$
\left(\sum_{e=0}^{m-1}\left(\begin{array}{c}
m-1 \\
e
\end{array}\right)\left(\begin{array}{c}
n-1 \\
e
\end{array}\right) z^{e}\right)^{2}=\left(\sum_{e=0}^{m-1}\left(\begin{array}{c}
m-1 \\
e
\end{array}\right)\left(\begin{array}{c}
n-1 \\
e
\end{array}\right) z^{m-1-e}\right)^{2} .
$$

Since both the polynomials inside the square brackets on the two sides of the above equality have positive leading coefficients, it follows that $R / I_{0}$ is Gorenstein if and only if $\left(\begin{array}{c}n-1 \\ e\end{array}\right)=\left(\begin{array}{c}n-1 \\ m-1-e\end{array}\right)$ for all $e=0,1, \ldots, m-1$. Since $1<m-1 \leq n-1$, the latter clearly holds if and only if $m=n$. 


\section{Acknowledgments}

Ghorpade is partially supported by Indo-Russian project INT/RFBR/P-114 from the Department of Science and Technology, Government of India and IRCC Award grant 12IRAWD009 from Indian Institute of Technology Bombay. Sethuraman was supported in part by NSF grants DMS-0700904 and CCF-1318260 during the preparation of the paper. He would like to thank the mathematics department at Indian Institute of Technology Bombay for its warm hospitality throughout the yearlong visit during some of which part of this work was done. Ghorpade would like to thank the mathematics department at California State University Northridge for the summer visit during which this collaboration took root. The work presented in this paper originated from the Master's thesis of Jonov at California State University Northridge and is a continuation of his earlier paper [Jonov 2011].

\section{References}

[Abhyankar 1988] S. S. Abhyankar, Enumerative combinatorics of Young tableaux, Monographs and Textbooks in Pure and Applied Mathematics 115, Marcel Dekker, New York, 1988. MR 89e:05011 Zbl 0643.05001

[Arbarello et al. 1985] E. Arbarello, M. Cornalba, P. A. Griffiths, and J. Harris, Geometry of algebraic curves, I, Grundlehren der Mathematischen Wissenschaften 267, Springer, New York, 1985. MR 86h:14019 Zbl 0559.14017

[Bruns and Conca 2003] W. Bruns and A. Conca, "Gröbner bases and determinantal ideals", pp. 9-66 in Commutative algebra, singularities and computer algebra (Sinaia, 2002), edited by J. Herzog and V. Vuletescu, NATO Sci. Ser. II Math. Phys. Chem. 115, Kluwer, Dordrecht, 2003. MR 2005a:13025 Zbl 1052.13010

[Bruns and Herzog 1993] W. Bruns and J. Herzog, Cohen-Macaulay rings, Cambridge Studies in Advanced Mathematics 39, Cambridge University Press, 1993. MR 95h:13020 Zbl 0788.13005

[Bruschek et al. 2011] C. Bruschek, H. Mourtada, and J. Schepers, "Arc spaces and RogersRamanujan identities", pp. 211-220 in 23rd International Conference on Formal Power Series and Algebraic Combinatorics (Reykjavik, 2011), Assoc. Discrete Math. Theor. Comput. Sci., Nancy, 2011. MR 2012m:14025

[Chaundy 1932] T. Chaundy, “The unrestricted plane partition”, Quart. J. Math., Oxf. Ser. 3 (1932), 76-80. Zbl 0004.09704

[Conca and Herzog 1994] A. Conca and J. Herzog, "On the Hilbert function of determinantal rings and their canonical module", Proc. Amer. Math. Soc. 122:3 (1994), 677-681. MR 95a:13016 Zbl 0823.13008

[Docampo 2013] R. Docampo, "Arcs on determinantal varieties", Trans. Amer. Math. Soc. 365:5 (2013), 2241-2269. MR 3020097 Zbl 1272.14014

[Ein and Mustaţă 2009] L. Ein and M. Mustaţă, "Jet schemes and singularities", pp. 505-546 in Algebraic geometry-Seattle 2005, II (Seattle, WA, 2005), edited by D. Abramovich et al., Proc. Sympos. Pure Math. 80, Amer. Math. Soc., Providence, RI, 2009. MR 2010h:14004 Zbl 1181.14019

[Galligo 1985] A. Galligo, "Computations of some Hilbert functions related with Schubert calculus", pp. 79-97 in Algebraic geometry (Sitges, Barcelona, 1983), edited by E. Casas-Alvero et al., Lecture Notes in Math. 1124, Springer, Berlin, 1985. MR 87j:14083 Zbl 0589.14039 
[Gessel and Viennot 1985] I. Gessel and G. Viennot, "Binomial determinants, paths, and hook length formulae", Adv. in Math. 58:3 (1985), 300-321. MR 87e:05008 Zbl 0579.05004

[Ghorpade 1994] S. R. Ghorpade, "Abhyankar's work on Young tableaux and some recent developments", pp. 215-249 in Algebraic geometry and its applications (West Lafayette, IN, 1990), edited by C. Bajaj, Springer, New York, 1994. MR 95h:05148 Zbl 0814.14046

[Ghorpade 1996] S. R. Ghorpade, "Young bitableaux, lattice paths and Hilbert functions", J. Statist. Plann. Inference 54:1 (1996), 55-66. MR 98c:05165 Zbl 0864.05088

[Ghorpade 2001] S. R. Ghorpade, "A note on Hodge's postulation formula for Schubert varieties", pp. 211-219 in Geometric and combinatorial aspects of commutative algebra (Messina, 1999), edited by J. Herzog and G. Restuccia, Lecture Notes in Pure and Appl. Math. 217, Dekker, New York, 2001. MR 2002d:14079 Zbl 0986.14028

[Ghorpade and Krattenthaler 2004] S. R. Ghorpade and C. Krattenthaler, "The Hilbert series of Pfaffian rings", pp. 337-356 in Algebra, arithmetic and geometry with applications (West Lafayette, IN, 2000), edited by C. Christensen et al., Springer, Berlin, 2004. MR 2005c:13010 Zbl 1083.13504

[Goward and Smith 2006] R. A. Goward, Jr. and K. E. Smith, "The jet scheme of a monomial scheme", Comm. Algebra 34:5 (2006), 1591-1598. MR 2007e:13037 Zbl 1120.14055

[Gräbe 1988] H.-G. Gräbe, Streckungsringe, Dissertation B, Erfurt/Mühlhausen, 1988.

[Herzog and Trung 1992] J. Herzog and N. V. Trung, "Gröbner bases and multiplicity of determinantal and Pfaffian ideals", Adv. Math. 96:1 (1992), 1-37. MR 94a:13012 Zbl 0778.13022

[Jonov 2011] B. Jonov, "Initial complex associated to a jet scheme of a determinantal variety", $J$. Pure Appl. Algebra 215:5 (2011), 806-811. MR 2011j:14103 Zbl 1219.13013

[Karlin and McGregor 1959] S. Karlin and J. McGregor, "Coincidence probabilities", Pacific J. Math. 9 (1959), 1141-1164. MR 22 \#5072 Zbl 0092.34503

[Košir and Sethuraman 2005a] T. Košir and B. A. Sethuraman, "Determinantal varieties over truncated polynomial rings”, J. Pure Appl. Algebra 195:1 (2005), 75-95. MR 2005h:13020 Zbl 1085.14043

[Košir and Sethuraman 2005b] T. Košir and B. A. Sethuraman, "A Groebner basis for the $2 \times 2$ determinantal ideal mod $t^{2}$," J. Algebra 292:1 (2005), 138-153. MR 2006f:13011 Zbl 1105.13009

[Krattenthaler 1995a] C. Krattenthaler, "Counting nonintersecting lattice paths with turns", Sém. Lothar. Combin. 34 (1995), Art. B34i, 17 pp. MR 97e:05018 Zbl 0855.05004

[Krattenthaler 1995b] C. Krattenthaler, The major counting of nonintersecting lattice paths and generating functions for tableaux, Mem. Amer. Math. Soc. 552, 1995. MR 95i:05109 Zbl 0830.05003

[Kulkarni 1996] D. M. Kulkarni, "Counting of paths and coefficients of the Hilbert polynomial of a determinantal ideal”, Discrete Math. 154:1-3 (1996), 141-151. MR 97f:13018 Zbl 0853.05005

[Lindström 1973] B. Lindström, "On the vector representations of induced matroids", Bull. London Math. Soc. 5 (1973), 85-90. MR 49 \#95 Zbl 0262.05018

[Liu 2002] Q. Liu, Algebraic geometry and arithmetic curves, Oxford Graduate Texts in Mathematics 6, Oxford University Press, Oxford, 2002. MR 2003g:14001 Zbl 0996.14005

[Modak 1992] M. R. Modak, "Combinatorial meaning of the coefficients of a Hilbert polynomial", Proc. Indian Acad. Sci. Math. Sci. 102:2 (1992), 93-123. MR 94e:13022 Zbl 0761.13006

[Mourtada 2011] H. Mourtada, "Jet schemes of toric surfaces", C. R. Math. Acad. Sci. Paris 349:9-10 (2011), 563-566. MR 2012e:14029 Zbl 1222.14031

[Mustaţă 2001] M. Mustaţă, "Jet schemes of locally complete intersection canonical singularities", Invent. Math. 145:3 (2001), 397-424. MR 2002f:14005 Zbl 1091.14004

[Mustaţă 2002] M. Mustaţă, "Singularities of pairs via jet schemes", J. Amer. Math. Soc. 15:3 (2002), 599-615. MR 2003b:14005 Zbl 0998.14009 
[Nash 1995] J. F. Nash, Jr., "Arc structure of singularities", Duke Math. J. 81:1 (1995), 31-38. MR 98f:14011 Zbl 0880.14010

[Sethuraman and Šivic 2009] B. A. Sethuraman and K. Šivic, "Jet schemes of the commuting matrix pairs scheme", Proc. Amer. Math. Soc. 137:12 (2009), 3953-3967. MR 2011b:14117 Zbl 1185.14046

[Smith and Weyman 2007] G. Smith and J. Weyman, "Resolutions of tangent schemes of determinantal ideals", private communication, 2007.

[Stanley 1978] R. P. Stanley, "Hilbert functions of graded algebras", Advances in Math. 28:1 (1978), 57-83. MR 58 \#5637 Zbl 0384.13012

[Svanes 1974] T. Svanes, "Coherent cohomology on Schubert subschemes of flag schemes and applications", Advances in Math. 14 (1974), 369-453. MR 54 \#7490 Zbl 0308.14008

[Yuen 2007a] C. Yuen, "Jet schemes of determinantal varieties", pp. 261-270 in Algebra, geometry and their interactions, edited by A. Corso et al., Contemp. Math. 448, Amer. Math. Soc., Providence, RI, 2007. MR 2009b:14093 Zbl 1142.14032

[Yuen 2007b] C. Yuen, "The multiplicity of jet schemes of a simple normal crossing divisor", Comm. Algebra 35:12 (2007), 3909-3911. MR 2008i:13051 Zbl 1127.14052

Received July 5, 2013.

SUDHIR R. GHORPADE

DEPARTMENT OF MATHEMATICS

INDIAN INSTITUTE OF TECHNOLOGY BOMBAY

POWAI, MUMBAI 400076

INDIA

srg@math.iitb.ac.in

BOYAN JONOV

DEPARTMENT OF MATHEMATICS

UNIVERSITY OF CALIFORNIA SANTA BARBARA

SANTA BARBARA, CA 93106

UNITED STATES

boyan@math.ucsb.edu

B. A. Sethuraman

DEPARTMENT OF MATHEMATICS

CALIFORNIA STATE UNIVERSITY NORTHRIDGE

NORTHRIDGE, CA 91330

UNITED STATES

al.sethuraman@csun.edu 


\title{
PACIFIC JOURNAL OF MATHEMATICS
}

\author{
msp.org/pjm
}

Founded in 1951 by E. F. Beckenbach (1906-1982) and F. Wolf (1904-1989)

\section{EDITORS}

Don Blasius (Managing Editor)

Department of Mathematics

University of California

Los Angeles, CA 90095-1555

blasius@math.ucla.edu

\author{
Paul Balmer \\ Department of Mathematics \\ University of California \\ Los Angeles, CA 90095-1555 \\ balmer@math.ucla.edu \\ Robert Finn \\ Department of Mathematics \\ Stanford University \\ Stanford, CA 94305-2125 \\ finn@math.stanford.edu \\ Sorin Popa \\ Department of Mathematics \\ University of California \\ Los Angeles, CA 90095-1555 \\ popa@math.ucla.edu
}

\author{
Vyjayanthi Chari \\ Department of Mathematics \\ University of California \\ Riverside, CA 92521-0135 \\ chari@math.ucr.edu \\ Kefeng Liu \\ Department of Mathematics \\ University of California \\ Los Angeles, CA 90095-1555 \\ liu@math.ucla.edu \\ Jie Qing \\ Department of Mathematics \\ University of California \\ Santa Cruz, CA 95064 \\ qing@ cats.ucsc.edu
}

\section{PRODUCTION}

Silvio Levy, Scientific Editor, production@msp.org

\section{SUPPORTING INSTITUTIONS}

ACADEMIA SINICA, TAIPEI

CALIFORNIA INST. OF TECHNOLOGY

INST. DE MATEMÁTICA PURA E APLICADA

KEIO UNIVERSITY

MATH. SCIENCES RESEARCH INSTITUTE

NEW MEXICO STATE UNIV.

OREGON STATE UNIV.

\author{
STANFORD UNIVERSITY \\ UNIV. OF BRITISH COLUMBIA \\ UNIV. OF CALIFORNIA, BERKELEY \\ UNIV. OF CALIFORNIA, DAVIS \\ UNIV. OF CALIFORNIA, LOS ANGELES \\ UNIV. OF CALIFORNIA, RIVERSIDE \\ UNIV. OF CALIFORNIA, SAN DIEGO \\ UNIV. OF CALIF., SANTA BARBARA
}

\author{
Daryl Cooper \\ Department of Mathematics \\ University of California \\ Santa Barbara, CA 93106-3080 \\ cooper@math.ucsb.edu \\ Jiang-Hua Lu \\ Department of Mathematics \\ The University of Hong Kong \\ Pokfulam Rd., Hong Kong \\ jhlu@maths.hku.hk \\ Paul Yang \\ Department of Mathematics \\ Princeton University \\ Princeton NJ 08544-1000 \\ yang@math.princeton.edu
}

These supporting institutions contribute to the cost of publication of this Journal, but they are not owners or publishers and have no responsibility for its contents or policies.

See inside back cover or msp.org/pjm for submission instructions.

The subscription price for 2014 is US $\$ 410 /$ year for the electronic version, and \$535/year for print and electronic.

Subscriptions, requests for back issues and changes of subscribers address should be sent to Pacific Journal of Mathematics, P.O. Box 4163, Berkeley, CA 94704-0163, U.S.A. The Pacific Journal of Mathematics is indexed by Mathematical Reviews, Zentralblatt MATH, PASCAL CNRS Index, Referativnyi Zhurnal, Current Mathematical Publications and Web of Knowledge (Science Citation Index).

The Pacific Journal of Mathematics (ISSN 0030-8730) at the University of California, c/o Department of Mathematics, 798 Evans Hall \#3840, Berkeley, CA 94720-3840, is published twelve times a year. Periodical rate postage paid at Berkeley, CA 94704, and additional mailing offices. POSTMASTER: send address changes to Pacific Journal of Mathematics, P.O. Box 4163, Berkeley, CA 94704-0163.

PJM peer review and production are managed by EditFLOW ${ }^{\circledR}$ from Mathematical Sciences Publishers.

\section{PUBLISHED BY}

\section{mathematical sciences publishers \\ nonprofit scientific publishing}

http://msp.org/

(C) 2014 Mathematical Sciences Publishers 


\section{PACIFIC JOURNAL OF MATHEMATICS}

Volume $272 \quad$ No. $1 \quad$ November 2014

Nonconcordant links with homology cobordant zero-framed surgery $\quad 1$ manifolds

JAE CHOON CHA and MARK POWELL

Certain self-homotopy equivalences on wedge products of Moore spaces

Ho Won CHOI and KeE Young LeE

Modular transformations involving the Mordell integral in Ramanujan's lost notebook

Youn-SEO CHOI

The $D$-topology for diffeological spaces

J. DANiEl Christensen, Gordon SinNAMON and ENXIN Wu

On the Atkin polynomials

AHMAD EL-Guindy and Mourad E. H. ISMAIL

Evolving convex curves to constant-width ones by a perimeter-preserving flow

LAIYUAN GaO and Shengliang PAN

Hilbert series of certain jet schemes of determinantal varieties

SudhiR R. GHORPADE, Boyan Jonov and B. A. SeTHuraman

On a Liu-Yau type inequality for surfaces

Oussama Hijazi, Sebastián Montiel and Simon Raulot

Nonlinear Euler sums

ISTVÁN MEZŐ

Boundary limits for fractional Poisson $a$-extensions of $L^{p}$ boundary functions 227 in a cone

LEI QIAO and TAO ZHAO

Jacobi-Trudi determinants and characters of minimal affinizations

STEVEN V SAM

Normal families of holomorphic mappings into complex projective space concerning shared hyperplanes

LiU Yang, Caiyun Fang and Xuecheng Pang 